\title{
COMPLEX AND INTEGRAL LAMINATED LATTICES
}

BY

J. H. CONWAY AND N. J. A. SLOANE

\begin{abstract}
In an earlier paper we studied real laminated lattices (or Z-modules) $\Lambda_{n}$, where $\Lambda_{1}$ is the lattice of even integers, and $\Lambda_{n}$ is obtained by stacking layers of a suitable $(n-1)$-dimensional lattice $\Lambda_{n-1}$ as densely as possible, keeping the same minimal norm. The present paper considers two generalizations: (a) complex and quaternionic lattices, obtained by replacing $\mathbf{Z}$-module by $J$-module, where $J$ may be the Eisenstein, Gaussian or Hurwitzian integers, etc., and (b) integral laminated lattices, in which $\Lambda_{n}$ is required to be an integral lattice with the prescribed minimal norm. This enables us to give a partial answer to a question of J. G. Thompson on integral lattices, and to explain some of the computer-generated results of Plesken and Pohst on this problem. Also a number of familiar lattices now arise in a canonical way. For example the Coxeter-Todd lattice is the 6-dimensional integral laminated lattice over $\mathbf{Z}[\omega]$ of minimal norm 2. The paper includes tables of the best real integral lattices in up to 24 dimensions.
\end{abstract}

\section{PART I. INTRODUCTION}

In our earlier paper [8] we analyzed the densest real lattices that can be built up by layers, starting with the one-dimensional lattice $\Lambda_{1}$ of even integers, and at the $n$th step stacking layers (or laminae), consisting of copies of a suitable $(n-1)$ dimensional lattice $\Lambda_{n-1}$, as close together as possible while keeping the same minimal norm. The resulting lattices we called laminated lattices $\Lambda_{n}$. A formal definition is given in $\$ 2.2$ below, and the main results of [8] are summarized in $\$ 2.3$.

In the present paper we consider two generalizations: (a) complex and quaternionic laminated lattices, and (b) integral laminations. For the first generalization we replace lattice ( $=\mathbf{Z}$-module) in the above definition by $J$-module, where $J$ is an appropriate ring of integers. We restrict ourselves to the following seven possibilities for $J$ : the rational integers $\mathbf{Z}$ (which of course is the case considered in [8]), the Eisenstein integers $Z[\omega]$, where $\omega=\frac{1}{2}(-1+\sqrt{-3})[13$, p. 112; 18, p. 188], the Gaussian integers $\mathbf{Z}[i]$, together with the rings $\mathbf{Z}[\lambda]$, $\mathbf{Z}[\eta]$ and $\mathbf{Z}[\nu]$, where

$$
\lambda=\frac{-1+\sqrt{-7}}{2}, \quad \eta=\sqrt{-2}, \quad \nu=\frac{-1+\sqrt{-11}}{2},
$$

and the Hurwitz quaternionic integers $\mathbf{H}_{\mathrm{z}}$ (defined below). A typical laminated $J$-lattice will be denoted by $\Lambda_{n, \zeta}$ if $J=\mathbf{Z}[\zeta]$, or by $\Lambda_{n, \mathbf{H}}$ if $J=\mathbf{H}_{\mathbf{Z}}$. These lattices are the subject of Part II. After certain preliminaries, the lattices $\Lambda_{n, \mathbf{H}}$ are investigated in $\S 2.6$ (see Theorem 4), $\Lambda_{n, \omega}, \Lambda_{n, i}$ and $\Lambda_{n, \eta}$ in $\$ 2.7$ (Theorems 5 and 6), and $\Lambda_{n, \lambda}$ in

Received by the editors December 8, 1982.

1980 Mathematics Subject Classification. Primary 10C05, 10E05, 52A43.

(C)1983 American Mathematical Society $0002-9947 / 83 \$ 1.00+\$ .25$ per page 
$\bar{\nu}$ belong to $J$ (the formal definition is in $\S 3.2$ ). A typical integral laminated ar $J$ of minimal norm $M$ will be denoted by $\Lambda_{n}[M]$ if $J=\mathbf{Z}$, by $\Lambda_{n}^{\zeta}$ [ $=\mathbf{Z}[\zeta]$, and by $\Lambda_{n}^{\mathbf{H}}[M]$ if $J=\mathbf{H}_{\mathbf{Z}}$. For the complex lattices the case $M=2$ st interesting, and we abbreviate $\Lambda_{n}^{\zeta}[2]$ to $\Lambda_{n}^{\zeta}$. The real lattices $\Lambda_{n}[M]$ are st Part III and the others in Part IV.

The reason we restrict ourselves to the five algebraic number rings ment ive is the following. If an algebraic number ring contains an irrational nber then it does not admit a discrete lattice. If the ring is not a principal nain, a lattice need not have an integral basis. This leaves the nine imag Idratic number rings of discriminants $-1,-2,-3,-7,-11,-19,-43,-6$; i3 [1. 351. The last four. however. contain no integers of norm 2. and so for 
lattice is $\Lambda_{12, \omega}(\$ 2.7)$, and a pair of interesting 3- and 4-dimensional lattices over $\mathbf{Z}[\lambda]$ arise as $\Lambda_{3}^{\lambda}$ and $\Lambda_{4, \lambda}(\$ \S 4.4,2.9)$.

(2) The method used to prove Theorem 10 (and described in $\$ 4.1$ ) could easily be extended to obtain a proof of the theorem that the root lattices $\Lambda_{0}, A_{1}, A_{2}, A_{3} \cong D_{3}$, $D_{4}, D_{5}, E_{6}, E_{7}, E_{8}($ see $[7,8])$ are the unique laminated lattices $\Lambda_{0}, \ldots, \Lambda_{8}$. The only extra step needed is a proof that the deep holes in $\Lambda_{0}, \ldots, E_{7}$ belong to the dual lattices $\Lambda_{0}^{*}, \ldots, E_{7}^{*}$. This can be supplied using Norton's technique [8, 29], although we do not give the details here. In [8] we deduced this theorem from the results of Blichfeldt [2] and Vetchinkin [37]. The new proof would make the determination of the $\Lambda_{n}$ for $n \leqslant 48$ in [8] independent of Blichfeldt and Vetchinkin's work (the inductive argument would then proceed one dimension at a time instead of in steps of 8 dimensions). Of course Theorem 3 of [8] still depends on [ 2 and 37].

Quaternions. $\mathbf{R}$ denotes the real numbers, $\mathbf{C}$ the complex numbers and $\mathbf{H}$ the quaternions. The inner product of two vectors $x, y$ is given by $x \cdot \bar{y}$, and the norm of a vector $x$ by $N(x)=x \cdot \bar{x}$. The Hurwitz integers in $\mathbf{H}$ are denoted by $\mathbf{H}_{\mathbf{z}}$, and consist of the quaternions $a+b i+c j+d k$, where $a, b, c, d$ are all in $\mathbf{Z}$ or all in $\mathbf{Z}+\frac{1}{2}[19]$. The 24 units of $\mathbf{H}_{\mathbf{z}}$ are $\pm 1, \pm i, \pm j, \pm k, \pm \omega, \pm \omega^{i}, \pm \omega^{j}, \pm \omega^{k}, \pm \bar{\omega}$, $\pm \bar{\omega}^{i}, \pm \bar{\omega}^{j}, \pm \bar{\omega}^{k}$, where

$$
\begin{aligned}
& \omega:=\frac{1}{2}(-1+i+j+k), \\
& \omega^{i}:=i^{-1} \omega i=j \omega=\omega k=\bar{\omega}+i=\frac{1}{2}(-1+i-j-k), \\
& \bar{\omega}^{i}:=i^{-1} \bar{\omega} i=-k \bar{\omega}=-\bar{\omega} j=\omega-i=\frac{1}{2}(-1-i+j+k),
\end{aligned}
$$

etc. As a multiplicative group this set of units is isomorphic to $2 \mathbf{A}_{4}$ (where $\mathbf{A}_{n}$ denotes the alternating group of degree $n$ ).

\section{PART II. COMPleX AND QUATERnionic LAMINATED latTiCES}

2.1. Real, complex and quaternionic lattices; terminology. The real, complex and quaternionic lattices we discuss in this paper are $J$-modules, where $J$ is one of the following seven rings of integers: $\mathbf{Z}, \mathbf{Z}[\zeta]$, where $\zeta$ is $\omega, i, \lambda, \eta$ or $\nu$ (see Part I), or $\mathbf{H}_{\mathbf{z}}$. In the case $J=\mathbf{H}_{\mathbf{z}}$ we take our lattices to be left $\mathbf{H}_{\mathbf{z}}$-modules [24, p. 9]. The seven rings are all principal ideal domains, and hence our lattices are free (i.e. have integral bases; compare [30, §81]). We shall call such a lattice a J-lattice.

A basis for a $J$-lattice $\Lambda$ consists of vectors $v_{1}, \ldots, v_{n}$ such that

$$
\Lambda=J v_{1}+J v_{2}+\cdots+J v_{n}
$$

which we abbreviate to $\Lambda=J\left\langle v_{1}, \ldots, v_{n}\right\rangle$. The smallest such $n$ is the dimension of $\Lambda$. Usually the subscript on the symbol for a lattice gives its dimension. If $G$, the Gram matrix of the lattice, is the $n \times n$ matrix with entries $v_{i} \cdot \bar{v}_{j}$ then the determinant of $\Lambda$ is det $\Lambda:=\operatorname{det} G$.

Suppose $\mathbf{K}$ is one of $\mathbf{R}, \mathbf{C}$ or $\mathbf{H}$, and $\Lambda_{n}, M_{n}$ are $J$-lattices, where $J \subseteq \mathbf{K}$. If $\Lambda_{n}$ and $M_{n}$ differ only by the appropriate kind of rotation and reflection, and possibly a scale factor, they are said to be isomorphic, and we write $\Lambda_{n} \cong M_{n}$. By an $r$-dimensional section of $\Lambda_{n}$ we mean an $r$-dimensional lattice $L_{r} \subseteq \mathbf{K}^{r} \subseteq \mathbf{K}^{n}$ such that $L_{r}=\Lambda_{n} \cap \mathbf{K}^{r}$. The dual lattice $\Lambda_{n}^{*}=\left\{x \in \mathbf{K}^{n}: x \cdot \bar{y} \in J\right.$ for all $\left.y \in \Lambda_{n}\right\} . \Lambda_{n}$ is integral if $\Lambda_{n} \subseteq \Lambda_{n}^{*}$, and unimodular if $\Lambda_{n}=\Lambda_{n}^{*}$. If $V$ is a subspace of $\mathbf{K}^{n}$, the 
orthogonal subspace $V^{\perp}=\left\{x \in \mathbf{K}^{n}: x \cdot \bar{y}=0\right.$ for all $\left.y \in V\right\}$. General references for lattices are $[12,21,26,28,30,34,38]$.

2.2. Laminated lattices. We can now give a precise statement of the laminated lattices problem. We first choose a number $M$, which is usually 4 . With $J$ as in $\S 2.1$, let us define a family $\mathbf{L}_{n}=\left\{L_{n}^{(1)}, \ldots, L_{n}^{\left(a_{n}\right)}\right\}$ of $J$-lattices inductively by specifying that $\mathbf{L}_{0}=\left\{\Lambda_{0}\right\}$, where $\Lambda_{0}$ is the one-point lattice, and that $\mathbf{L}_{n+1}=$ $\left\{L_{n+1}^{(1)}, \ldots, L_{n+1}^{\left(a_{n+1}\right)}\right\}$ consists of all $J$-lattices $L_{n+1}$ satisfying

(i) $L_{n+1}$ has minimal norm $M$,

(ii) $L_{n+1}$ contains at least one $L_{n}^{(i)}, 1 \leqslant i \leqslant a_{n}$, and

(iii) $L_{n+1}$ has the minimal possible determinant subject to (i) and (ii).

It is clear that the only effect of changing $M$ is to change the scale of all the lattices. Usually we take $M=4$, since on this scale nonoverlapping spheres of radius 1 can be drawn around the lattice points, the Leech lattice has determinant 1 , and the notation is consistent with [8].

In the case when $J=\mathbf{Z}$, a typical lattice of $\mathbf{L}_{n}$ is in $\mathbf{R}^{n}$, is called a real laminated lattice, and will be denoted by $\Lambda_{n}$ (as in [8]). When necessary superscripts are used to distinguish between different $\Lambda_{n}$ 's.

When $J=\mathbf{Z}[\zeta]$, the lattices of $\mathbf{L}_{n}$ are in $\mathbf{C}^{n}$, are called complex laminated lattices, and will be denoted by $\Lambda_{n, \xi}$. When $J=\mathbf{H}_{\mathbf{Z}}$ they are in $\mathbf{H}^{n}$ and are denoted by $\Lambda_{n, \mathbf{H}}$ (and in general by $\Lambda_{n, J}$ ). Our problem is to find (as far as possible) the lattices $\Lambda_{n, J}$. There is always a unique $\Lambda_{1, J}$, which is a scaled copy of $J$ itself.

2.3. Real laminated lattices. The case $J=\mathbf{Z}$ was extensively studied in [8], and the following theorem was proved.

TheOREM 2. For $0 \leqslant n \leqslant 48$ the determinant $\lambda_{n}$ of any real laminated lattice $\Lambda_{n}$ (with minimal norm $M=4$ ) is equal to

$$
\begin{array}{ll}
2^{n} c_{n} & \text { for } 0 \leqslant n \leqslant 8, \\
2^{8} c_{n-8} & \text { for } 8 \leqslant n \leqslant 16, \\
\lambda_{24-n} & \text { for } 12 \leqslant n \leqslant 24, \\
2^{24-n} \lambda_{n-24} & \text { for } 24 \leqslant n \leqslant 48,
\end{array}
$$

where $c_{n}$ is given by:

$$
\begin{array}{llllllllll}
n & 0 & 1 & 2 & 3 & 4 & 5 & 6 & 7 & 8 \\
c_{n} & 1 & 2 & 3 & 4 & 4 & 4 & 3 & 2 & 1
\end{array}
$$

(See Table I of [8]. The values of $\lambda_{n}$ may also be found in the column headed $\omega$ in Table I below.) All $\Lambda_{n}$ for $n \leqslant 24$ are shown in Figure 1.

For $n \leqslant 8$ the $\Lambda_{n}$ are better known by the following names [7, 28]:

$$
\begin{gathered}
\Lambda_{0}, \Lambda_{1} \cong \mathbf{Z} \cong A_{1}, \quad \Lambda_{2} \cong A_{2}, \quad \Lambda_{3} \cong A_{3} \cong D_{3}, \quad \Lambda_{4} \cong D_{4}, \\
\Lambda_{5} \cong D_{5}, \quad \Lambda_{6} \cong E_{6}, \quad \Lambda_{7} \cong E_{7}, \quad \Lambda_{8} \cong E_{8} .
\end{gathered}
$$

For $25 \leqslant n \leqslant 48$ we constructed one particular sequence of $\Lambda_{n}$ 's in [8] by gluing the Leech lattice $\Lambda_{24}$ to itself. We denote this sequence by $\Lambda_{n}^{(a)}(25 \leqslant n \leqslant 48)$. There 
are however many laminated lattices in these dimensions (see [8]). The particular sequence

$$
\Lambda_{0}, \Lambda_{4}, \Lambda_{8}, \Lambda_{12}^{\max }, \Lambda_{16}, \Lambda_{20}, \Lambda_{24}, \Lambda_{28}^{(a)}, \Lambda_{32}^{(a)}, \ldots, \Lambda_{48}^{(a)}
$$

will be used later in the paper.

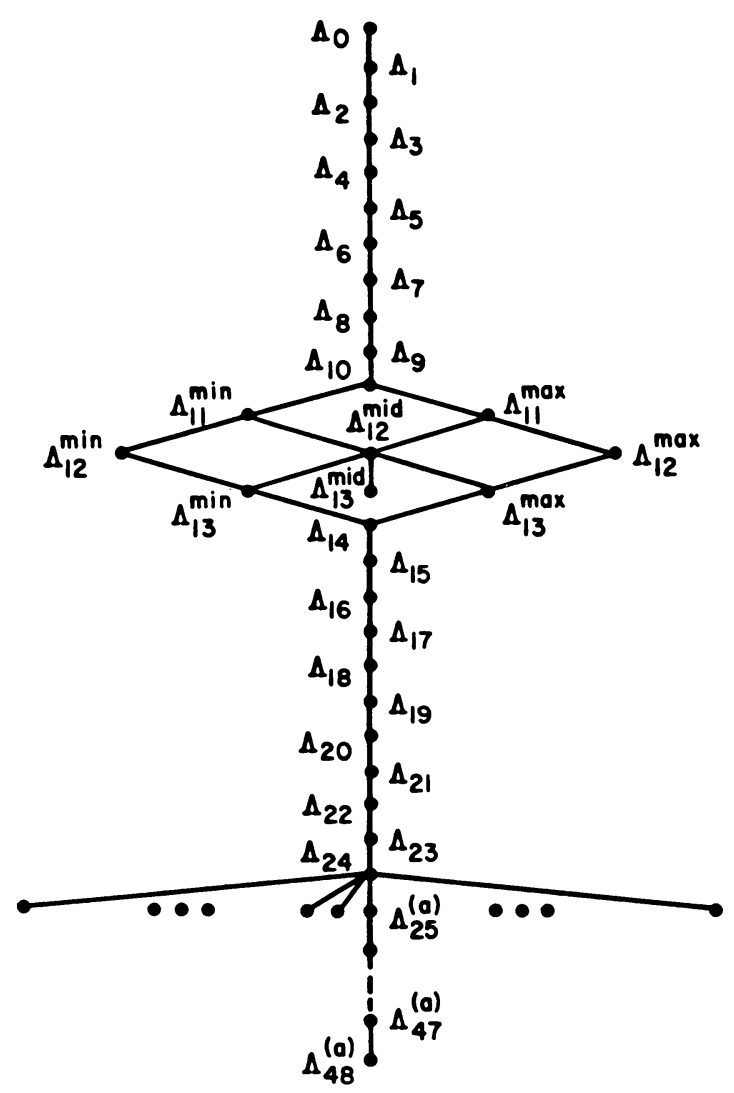

FIGURE 1. Real laminated lattices $\Lambda_{n}$ for $n \leqslant 48$ (from [8]). All $\Lambda_{n}$ are shown for $n \leqslant 24$, while there are 23 inequivalent $\Lambda_{25}$ 's. $\Lambda_{24}$ is the Leech lattice.

2.4. Relations between real, complex and quaternionic lattices. If $\Lambda \subseteq \mathbf{C}^{n}$ is a complex $J$-lattice, with $J=\mathbf{Z}[\zeta]$, there is a corresponding real lattice $\Lambda_{\text {real }}$ in $\mathbf{R}^{2 n}$, consisting of the vectors

$$
\left(\operatorname{Re}\left(x_{1}\right), \operatorname{Im}\left(x_{1}\right), \operatorname{Re}\left(x_{2}\right), \ldots, \operatorname{Im}\left(x_{n}\right)\right)
$$

for $\left(x_{1}, x_{2}, \ldots, x_{n}\right) \in \Lambda$. Also (see [34])

$$
\operatorname{det} \Lambda_{\text {real }}=\partial^{n} 4^{-n}(\operatorname{det} \Lambda)^{2} \text {, }
$$

where $\partial$, the absolute value of the discriminant of $J$, is equal to $3,4,7,8,11$ if $\zeta$ is $\omega$,

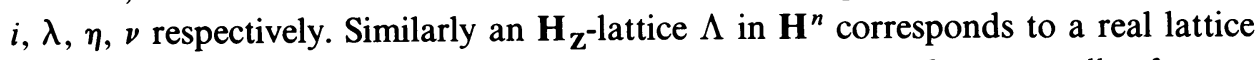
$\Lambda_{\text {real }}$ in $\mathbf{R}^{4 n}$, obtained by replacing every component $a+b i+c j+c k$ of every vector in $\Lambda$ by $\ldots a, b, c, d, \ldots$ In this case

$$
\operatorname{det} \Lambda_{\text {real }}=4^{-n}(\operatorname{det} \Lambda)^{4} \text {. }
$$


Let $L_{\pi}$ denote left multiplication of vectors in an $\mathbf{H}_{\mathbf{z}}$-lattice $\Lambda$ by $\pi$, where $\pi$ is any of the 24 units in $\mathbf{H}_{\mathbf{z}}$. Then, if $\pi \neq 1, L_{\pi}$ is a fixed-point free automorphism of $\Lambda$, i.e. only the origin is fixed by $L_{\pi}$. Furthermore $L_{\pi}$ induces a fixed-point free automorphism of $\Lambda_{\text {real }}$. Conversely, if $L$ is a real lattice in $\mathbf{R}^{4 n}$ which is a left $\left(2 \mathbf{A}_{4}\right)$-module, and every nontrivial element of $2 \mathbf{A}_{4}$ acts in a fixed-point free manner, then $L$ may be regarded as an $\mathbf{H}_{\mathbf{z}}$-lattice in $\mathbf{H}^{n}$. Similar assertions can be made for the other choices of $J$. For example a real lattice in $\mathbf{R}^{2 n}$ with a distinguished fixed-point free automorphism of order 3 can be regarded as a $\mathbf{Z}[\omega]$-lattice in $\mathbf{C}^{n}$.

Finally we note that $\mathbf{H}_{\mathbf{Z}}$ contains (in many ways) copies of $\mathbf{Z}[\omega], \mathbf{Z}[i]$ and $\mathbf{Z}[\eta] \cong \mathbf{Z}[j+k]$ (see Figure 2). Therefore an $\mathbf{H}_{\mathbf{Z}}$-lattice may also be regarded for example as a $\mathbf{Z}[\omega]$-lattice.

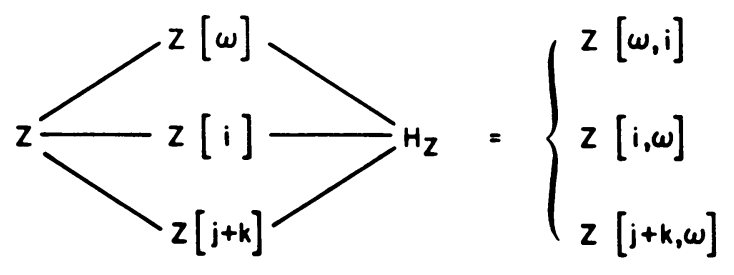

FIGURE 2. Inclusions among the rings of integers.

2.5. A useful theorem. The following theorem will make it easy to use the results of [8]. For it enables us to establish that a lattice $L_{r n+s}$ is a laminated lattice $\Lambda_{r n+s}$, without having to check that it contains laminated lattices of every lower dimension.

THEOREM 3. Let $r=1,2,4$ or $8,0 \leqslant s \leqslant r$, and $r n+s \leqslant 48$. Then any real $(r n+s)$-dimensional lattice $L_{r n+s}$ of minimal norm $M$ that contains a laminated lattice $\Lambda_{r n}$, and which satisfies $\operatorname{det} L_{r n+s} \leqslant \operatorname{det} \Lambda_{r n+s}$, is one of the lattices $\Lambda_{r n+s}$.

Proof. In [8, Theorem 3] it was shown that

$$
\operatorname{det} L_{r n+s} \geqslant \lambda_{r n} \lambda_{s}\left(\frac{M-h^{2}}{M}\right)^{s},
$$

where $\lambda_{k}=\operatorname{det} \Lambda_{k}$ and $h$ is the covering radius of $\Lambda_{r n}$. However it can be checked that in all the cases $r=1,2,4$, or $8,0 \leqslant s \leqslant r, r n+s \leqslant 48$, equality holds in (7). The argument used to prove Theorem 3 in [8] then establishes the result.

2.6. Quaternionic laminations. The following theorem describes the quaternionic laminated lattices $\Lambda_{n, \mathbf{H}}$ for $n \leqslant 12$.

THEOREM 4. The lattices (4) can be made into $\mathbf{H}_{\mathbf{z}}$-lattices, and in fact are quaternionic laminated lattices $\Lambda_{n, \mathrm{H}}$. Conversely any $\Lambda_{n, \mathrm{H}}$ for $0 \leqslant n \leqslant 12$ can be obtained by making some $\Lambda_{4 n}$ into an $\mathbf{H}_{\mathbf{z}}$-lattice.

Proof. The first step is to make the lattices (4) into left $\mathbf{H}_{\mathbf{z}}$-modules. For $n \leqslant 6$ they are sublattices of the Leech lattice $\Lambda_{24}$. As is customary we use MOG (Miracle Octad Generator) coordinates for vectors in $\mathbf{R}^{24}$, following $[5,16,17]$ and especially 
$[6,8]$. MOG coordinates in $\mathbf{R}^{24}$ can then be represented by quaternionic coordinates in $\mathbf{H}^{6}$ via the diagram:

\begin{tabular}{|ll|ll|ll|}
\hline 1 & 1 & 1 & 1 & 1 & 1 \\
$k$ & $j$ & $k$ & $j$ & $k$ & $j$ \\
\hline$i$ & $k$ & $i$ & $k$ & $i$ & $k$ \\
$j$ & $i$ & $j$ & $i$ & $j$ & $i$ \\
\hline
\end{tabular}

For example the Leech vector

\begin{tabular}{|ll|ll|ll|}
\hline 4 & 0 & 0 & 0 & 0 & 0 \\
4 & 0 & 0 & 0 & 0 & 0 \\
\hline 0 & 0 & 0 & 0 & 0 & 0 \\
0 & 0 & 0 & 0 & 0 & 0 \\
\hline
\end{tabular}

is represented by the quaternionic vector

$$
\frac{1}{\sqrt{8}}(4+4 k, 0,0,0,0,0) \text {. }
$$

(By convention it is necessary to divide MOG coordinates by $\sqrt{8}$ to make the minimal norm equal to 4 .)

When the Leech lattice is represented in $\mathbf{H}^{6}$ in this way, it is fixed under both left multiplication $L_{\pi}$ and right multiplication $R_{\pi}$ by units $\pi \in \mathbf{H}_{\mathbf{z}}$, and so becomes a two-sided $\mathbf{H}_{\mathbf{z}}$-module. The sublattices $\Lambda_{0}, \Lambda_{4}, \Lambda_{8}, \Lambda_{12}^{\max }, \Lambda_{16}$ and $\Lambda_{20}$ are defined in Figure 4 of [8]. They are also fixed under the maps $L_{\pi}$ and $R_{\pi}$, and hence are two-sided $\mathbf{H}_{\mathbf{z}}$-modules.

To make $\Lambda_{28}^{(a)}, \ldots, \Lambda_{48}^{(a)}$ into left $\mathbf{H}_{\mathbf{z}}$-modules we observe that, instead of the map $\theta$ used to construct them in $\S$ VII of [8], we could equally well have used $\theta^{\prime}:=$ $\left(1+R_{i}\right) / 2$. Since $\theta^{\prime}$ commutes with the maps $L_{\pi}$, these lattices can be regarded as left $\mathbf{H}_{\mathbf{z}}$-modules.

To show that these lattices are $\Lambda_{n, \mathbf{H}}$ 's we use induction on $n$. Suppose that a lattice $\Lambda_{4 n}$ mentioned in (4), when regarded as an $\mathbf{H}_{\mathbf{Z}}$-module, is a $\Lambda_{n, \mathbf{H}}$, for $0 \leqslant n \leqslant 11$. Consider any $\Lambda_{n+1, \mathbf{H}}$ containing this $\Lambda_{n, \mathbf{H}}$. Let $L_{4 n+4}$ be the real version of $\Lambda_{n+1, \mathbf{H}}$. Since $L_{4 n+4} \supseteq \Lambda_{4 n}$, by Theorem $3 L_{4 n+4}$ is a $\Lambda_{4 n+4}$. Therefore the lattices (4) are $\Lambda_{n, \mathbf{H}}$ 's, and the same argument also establishes the converse. This completes the proof of Theorem 4 .

2.7. $\mathbf{Z}[\omega], \mathbf{Z}[i]$, and $\mathbf{Z}[\eta]$-laminations. A laminated lattice $\Lambda_{n+1, J}$ (for any $J$ ) is constructed from a $\Lambda_{n, J}$ by adjoining a vector $v$, say, of norm $M$. We may write

$$
v=h+t, \quad N(h)+N(t)=M,
$$


where $h$ is in the space of $\Lambda_{n, J}$ and $t \in \Lambda_{n, J}^{\perp}$. Often $h$ is a deep hole in $\Lambda_{n, J}$, as in [8]. A typical layer of $\Lambda_{n+1, J}$ consists of the points

$$
\Lambda^{(\alpha)}:=\Lambda_{n, J}+\alpha v, \quad \alpha \in J .
$$

Since

$$
\operatorname{det} \Lambda_{n+1, J}=N(t) \operatorname{det} \Lambda_{n, J},
$$

we must choose $N(t)$ as small as possible (and $N(h)$ as large as possible) while keeping the minimal norm of $\Lambda_{n+1, J}$ equal to $M$. A necessary and sufficient condition for $\Lambda_{n+1, J}$ to have minimal norm $M$ is that the squared distance between $\Lambda_{n, J}$ and $\Lambda^{(\alpha)}$ is at least $M$, for all $\alpha \in J$, or in other words that

$$
N\left(\alpha h-\Lambda_{n, J}\right) \geqslant M-N(\alpha)(M-N(h)), \quad \text { all } \alpha \in J .
$$

This will obviously be true for all sufficiently large $\alpha$. We say that $v$ (or $h$ ) is $J$-acceptable if (9) holds.

We now consider the case when $J$ is one of the three rings $\mathbf{Z}[\omega], \mathbf{Z}[i]$ or $\mathbf{Z}[\eta]$ contained in $\mathbf{H}_{\mathbf{z}}$ (see Figure 2), and as usual take $M=4$.

TheOREM 5. Let $J$ be $\mathbf{Z}[\omega], \mathbf{Z}[i]$, or $\mathbf{Z}[\eta]$. For $0 \leqslant n \leqslant 12$ let $L_{2 n, J}$ be obtained by giving the $\Lambda_{4 n}$ in (4) the structure of J-lattices. Let $L_{2 n+1, J}=J\left\langle L_{2 n, J}, v\right\rangle$, where $v$ is such that $\Lambda_{4 n+1}=\mathbf{Z}\left\langle\Lambda_{4 n}, v\right\rangle$. Then $L_{m, J}(0 \leqslant m \leqslant 24)$ is a complex laminated lattice $\Lambda_{m, J}$, and has the determinant given in Table I. Conversely any $\Lambda_{2 n, J}$ for $0 \leqslant n \leqslant 12$ can be obtained by making some $\Lambda_{4 n}$ into a J-lattice

For $\Lambda_{4, i}$ see (27) in $\$ 4.3$.

Proof. We have already seen that the lattices $\Lambda_{4 n}$ in (4) are $\mathbf{H}_{\mathbf{z}}$-lattices, and therefore a fortiori are $J$-lattices for these $J$ 's. The results of $\$ 2.6$ imply that in these $\Lambda_{4 n}$ the best vector $v$ that is $\mathbf{Z}$-acceptable is also $\mathbf{H}_{\mathbf{z}}$-acceptable, and therefore $J$-acceptable. So if $L_{2 n, J}$ is a $\Lambda_{2 n, J}, L_{2 n+1, J}$ is a $\Lambda_{2 n+1, J}$ (for we are already using the best vector $v$ that we could hope to use). Since $L_{2 n+1, J}$ can be $J$-extended to $L_{2 n+2, J}$, Theorem 3 implies that $L_{2 n+2, J}$ is a $\Lambda_{2 n+2, J}$. For if not the real form of $\Lambda_{2 n+2, J}$ is a $(4 n+4)$-dimensional lattice containing $\Lambda_{4 n}$ and with smaller determinant than $\Lambda_{4 n+4}$, which is impossible. This proves that the $L_{m, J}$ are $\Lambda_{m, J}$. The converse also follows immediately from Theorem 3 .

The determinants are found as follows. If $\Lambda_{n+1, J}=J\left\langle\Lambda_{n, J}, v\right\rangle$, we define

$$
\Lambda_{n+1 / 2, J}=\mathbf{Z}\left\langle\Lambda_{n, J}, v\right\rangle \text {. }
$$

Then we have

$$
\begin{gathered}
\operatorname{det}\left(\Lambda_{2 n, J}\right)_{\text {real }}=\operatorname{det} \Lambda_{4 n}=\lambda_{4 n}, \\
\operatorname{det}\left(\Lambda_{n+1 / 2, J}\right)_{\text {real }}=N(t) \operatorname{det}\left(\Lambda_{n, J}\right)_{\text {real }}, \\
\operatorname{det}\left(\Lambda_{n+1, J}\right)_{\text {real }}=(\partial / 4) N(t)^{2} \operatorname{det}\left(\Lambda_{n, J}\right)_{\text {real }}
\end{gathered}
$$

((13) follows from (5) and (8)). The entries in Table I can now be computed using the values of $N(t)$ for $\Lambda_{4 n}$ given in [8, Table I]. This completes the proof of Theorem 5 . 
TABle I.

Determinants of complex laminated lattices $\Lambda_{m, \zeta}$ for $\zeta=\omega, i, \lambda, \eta$ and $0 \leqslant m \leqslant 24$, on the scale at which the minimal norm is 4 . The entries give $\operatorname{det}\left(\Lambda_{m, \xi}\right)_{\text {real }}$ and the real dimension $n=2 m$. The intermediate entries give $\operatorname{det}\left(\Lambda_{m+1 / 2, \xi}\right)_{\text {real }}$ in $n=2 m$ +1 real dimensions, where $\Lambda_{m+1 / 2, \zeta}=\mathbf{Z}\left\langle\Lambda_{m, \zeta}, v\right\rangle$ if $\Lambda_{m+1, \zeta}=J\left\langle\Lambda_{m, \zeta}, v\right\rangle$. The table may be extended to 48 real dimensions by the formula $\operatorname{det}\left(\Lambda_{n / 2+12, \xi}\right)_{\text {real }}=$ $2^{-n} \operatorname{det}\left(\Lambda_{n / 2, \xi}\right)_{\text {real }}$ for $24 \leqslant n \leqslant 48$. The column headed by $\omega$ also gives the values of $\lambda_{n}=\operatorname{det} \Lambda_{n}$ (see Theorem 2).

\begin{tabular}{|r|r|r|r|r|}
\hline$n$ & \multicolumn{1}{|c|}{$\omega$} & \multicolumn{1}{|c|}{$i$} & \multicolumn{1}{c|}{$\lambda$} & \multicolumn{1}{|c|}{$\eta$} \\
\hline \hline 0 & 1 & 1 & 1 & 1 \\
1 & 4 & 4 & 4 & 4 \\
2 & 12 & 16 & 28 & 32 \\
3 & 32 & 32 & 48 & 32 \\
4 & 64 & 64 & 144 & 64 \\
5 & 128 & 128 & 192 & 128 \\
6 & 192 & 256 & 448 & 512 \\
7 & 256 & 256 & 256 & 256 \\
8 & 256 & 256 & 256 & 256 \\
9 & 512 & 512 & 512 & 512 \\
10 & 768 & 1024 & 1792 & 2048 \\
11 & 1024 & 1024 & 1536 & 1024 \\
12 & 1024 & 1024 & 2304 & 1024 \\
13 & 1024 & 1024 & 1536 & 1024 \\
14 & 768 & 1024 & 1792 & 2048 \\
15 & 512 & 512 & 512 & 512 \\
16 & 256 & 256 & 256 & 256 \\
17 & 256 & 256 & 256 & 256 \\
18 & 192 & 256 & 448 & 512 \\
19 & 128 & 128 & 192 & 128 \\
20 & 64 & 64 & 144 & 64 \\
21 & 32 & 32 & 48 & 32 \\
22 & 12 & 16 & 28 & 32 \\
23 & 4 & 4 & 4 & 4 \\
24 & 1 & 1 & 1 & 1 \\
\hline
\end{tabular}

We note that in general $\left(\Lambda_{n+1 / 2, J}\right)_{\text {real }} \neq \Lambda_{2 n+1}$ and $\left(\Lambda_{2 n+1, J}\right)_{\text {real }} \neq \Lambda_{4 n+2}$.

The case $J=\mathbf{Z}[\omega]$ is particularly simple. We observe from Table I that, for every $m \leqslant 24$, the real form of $\Lambda_{m, \omega}$ has the same determinant as $\Lambda_{2 m}$, and therefore by Theorem 3 is a $\Lambda_{2 m}$. Of the $\Lambda_{2 m}$ 's for $2 m \leqslant 24$, it is easy to check that all except $\Lambda_{12}^{\text {mid }}$ can be made into $\mathbf{Z}[\omega]$-lattices, in an essentially unique way. On the other hand $\Lambda_{12}^{\text {mid }}$ has contact number 632 , which is not divisible by 3 , and so $\Lambda_{12}^{\text {mid }}$ is not a $\mathbf{Z}[\omega]$-lattice. We have therefore established the following result. 


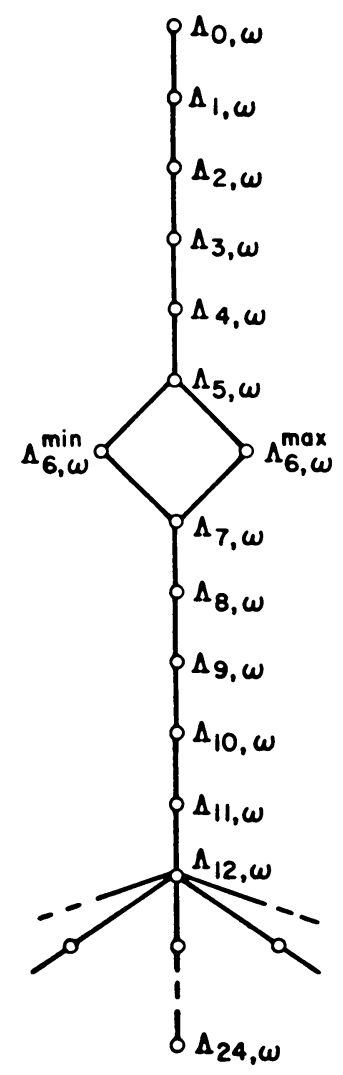

FIGURE 3. All complex laminated lattices $\Lambda_{n, \omega}$ for $n \leqslant 12$.

TheOREM 6. All the complex laminated lattices $\Lambda_{n, \omega}$ for $n \leqslant 12$ are shown in the inclusion lattice displayed in Figure 3.

Of course $\left(\Lambda_{12, \omega}\right)_{\text {real }} \cong \Lambda_{24}$, and $\Lambda_{12, \omega}$ is sometimes called the complex Leech lattice $[4,22,23,34,39]$. Its automorphism group is the six-fold cover of the Suzuki group.

2.8. Diagrams for minimal vectors. The minimal vectors of $\Lambda_{n+1, J}$ (for any $J$ ) correspond to the cases where equality holds in (9). If $J$ is complex there is a convenient way of representing the minimal vectors of $\Lambda_{2, J}$ diagrammatically. In this section we take $M=1$, so that $\Lambda_{1, J}=J$. Then $\Lambda_{2, J}=J\langle u, v\rangle$, where $u=(1,0)$, $v=(h, t), N(v)=1$, and $N(h)$ is as large as possible subject to (9).

The minimal vectors of $\Lambda_{2, J}$ have the form $\beta v-\alpha u=(\beta h-\alpha, \beta t)$, for $\alpha, \beta \in J$, and in the diagram are represented by thick arrows pointing from $\beta$ (indicated by a small circle) to $\alpha$. We have already seen (in Theorem 6) that there is a unique $\Lambda_{2, \omega}$, and it is not hard to prove that $\Lambda_{2, i}, \Lambda_{2, \lambda}$ and $\Lambda_{2, \eta}$ are also essentially unique. The diagrams for the minimal vectors of these lattices are shown in Figure 4. Consider for example Figure 4(d), which represents $\Lambda_{2, \eta}=\langle(1,0),(h, t)\rangle$, where $h=\frac{1}{2}(\eta-1)$, 
$N(h)=\frac{3}{4}$, and $t=\frac{1}{2}$. The minimal vectors have the form $\left(\frac{1}{2} \beta(\eta-1)-\alpha, \frac{1}{2} \beta\right)$, typical examples being

$$
\begin{array}{cl}
( \pm 1,0) & \begin{array}{l}
\text { represented by the two long horizontal arrows } \\
\text { leading from } \beta h=0 \text { to } \alpha= \pm 1,
\end{array} \\
\pm\left(h-x, \frac{1}{2}\right) & \begin{array}{l}
\text { where } x \text { is one of the four lattice points } 0, \eta \\
2 h=\eta-1,-1 \text { closest to } h, \text { represented by the } \\
\text { two crosses originating at } \beta h= \pm h, \text { and }
\end{array} \\
(0, \pm 1) \quad & \begin{array}{l}
\text { represented by the two curved arrows pointing } \\
\text { from } \beta h= \pm 2 h \text { to } \alpha=\beta h .
\end{array}
\end{array}
$$

(a)

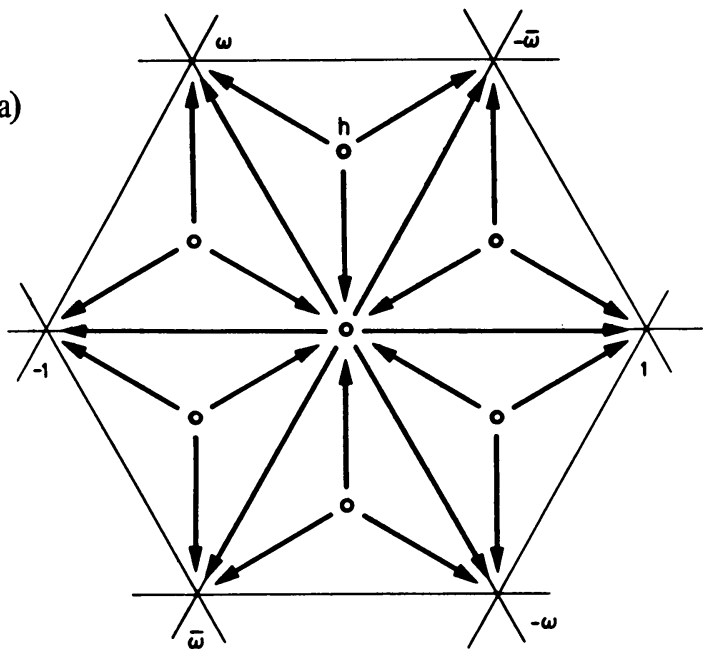

(b)

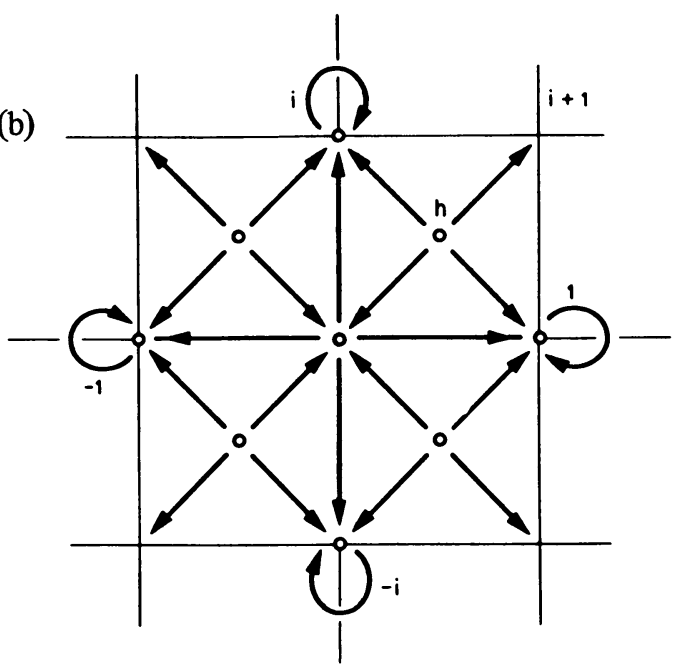

FIGURE 4. Diagrams representing the minimal vectors of (a) $\Lambda_{2, \omega}$, (b) $\Lambda_{2, i}$, (c) $\Lambda_{2, \lambda}$ and (d) $\Lambda_{2, \eta}$. Each thick arrow represents a minimal vector (see text). 


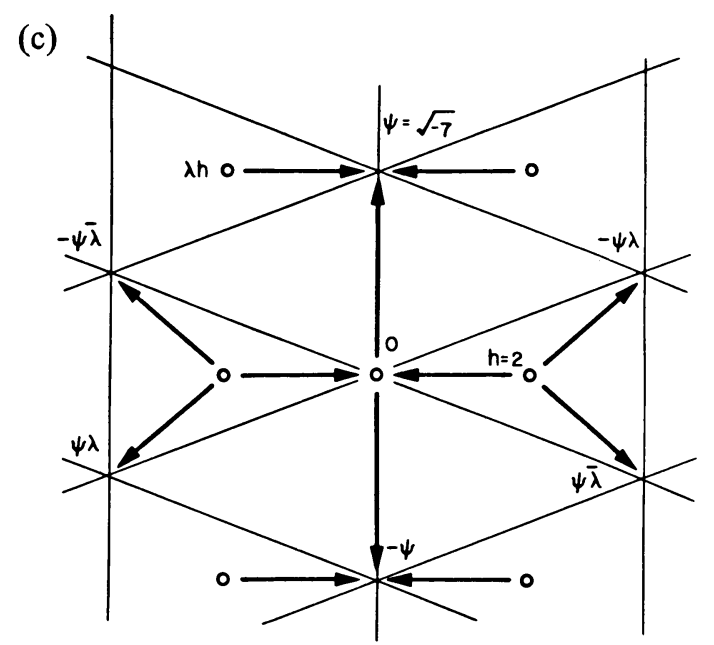

(d)

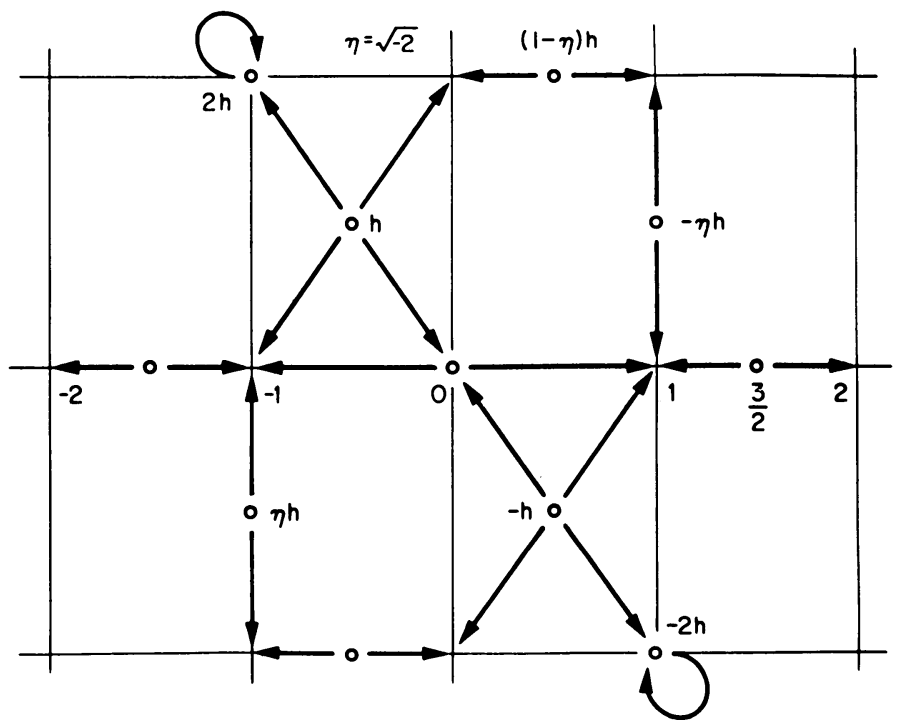

Figure 4. (Continued)

There are 24 arrows, so the contact number is 24 . In fact $\Lambda_{2, \omega}, \Lambda_{2, i}$ and $\Lambda_{2, \eta}$ all have contact number 24 (since they are complex versions of $\Lambda_{4}$, by Theorem 5), while $\Lambda_{2, \lambda}$ has contact number 12 .

2.9. $\mathbf{Z}[\lambda]$-laminations. In this section we study the case $J=\mathbf{Z}[\lambda]$, where $\lambda=$ $\frac{1}{2}(-1+\sqrt{-7}), \lambda^{2}+\lambda+2=0$. It is convenient when constructing these lattices to set the minimal norm $M=7$. We also write $\psi:=\lambda-\bar{\lambda}=\sqrt{-7}$.

THEOREM 7. (i) The lattices $\Lambda_{r, \lambda}$ for $0 \leqslant r \leqslant 3$ are unique (see (14), (15)).

(ii) $\Lambda_{0}, \Lambda_{8}, \Lambda_{16}, \Lambda_{24}, \Lambda_{32}^{(a)}, \Lambda_{40}^{(a)}$ and $\Lambda_{48}^{(a)}$ can be made into $\mathrm{Z}[\lambda]$-lattices, and are $\Lambda_{4 n, \lambda}$ 's. Conversely any $\Lambda_{4 n, \lambda}$ for $0 \leqslant n \leqslant 6$ can be obtained by making some $\Lambda_{8 n}$ into a $\mathbf{Z}[\lambda]$-lattice.

(ii) Any $\Lambda_{4 n+r, \lambda}$ for $0 \leqslant r \leqslant 4,0 \leqslant n \leqslant 11$ may be obtained by projecting a $\Lambda_{4 n+4, \lambda}$ onto a $\Lambda_{4 n, \lambda}^{\perp}$, which produces a scaled copy of $a \Lambda_{4, \lambda}$, and taking the inverse image of $a$ $\Lambda_{r, \lambda}$ in this $\Lambda_{4, \lambda}$. 
(iv) The determinant of $\Lambda_{m, \lambda}, 0 \leqslant m \leqslant 24$, when scaled so that the minimal norm is 4, is shown in Table I.

Proof. We take $\Lambda_{1, \lambda}=\psi J$ as shown in Figure 4(c). There is a unique type of deep hole in $\Lambda_{1, \lambda}$, which we take to be $h=2$. Then

$$
\Lambda_{2, \lambda}=J\langle u=(\sqrt{-7}, 0), v=(2, \sqrt{3})\rangle .
$$

This lattice has the 12 minimal vectors

$$
\pm\{u, \bar{\lambda} u-v, \lambda u+v, v, \lambda v-u, \bar{\lambda} v+u\}
$$

which are also shown in Figure 4(c). Furthermore $\left(\Lambda_{2, \lambda}\right)_{\text {real }} \cong A_{2} \oplus A_{2}$, where the first three vectors in (15) span one copy of $A_{2}$, the last three the other copy. Typical deep holes in these $A_{2}$ 's are

$$
x=\frac{2 v+\psi u}{3}, \quad y=\frac{2 u-\psi v}{3}
$$

respectively. It is not difficult to find the automorphism group of $\Lambda_{2, \lambda}$, which is a group of order 12 isomorphic to $\langle 2,2,3\rangle$ [14, Table I], and from this to show that all the deep holes in $\Lambda_{2, \lambda}$ yield the same lattice $\Lambda_{3, \lambda}$, given by $\Lambda_{3, \lambda}=J\langle u, v, w\rangle$, where

$$
w=\left(x+y, \sqrt{\frac{7}{3}}\right)=\left(\frac{(2+\psi) u+(2-\psi) v}{3}, \sqrt{\frac{7}{3}}\right) .
$$

Nicer coordinates may be obtained by replacing $u, v, w$ by the vectors

$$
U=\left(\lambda^{2}, 1,-\bar{\lambda}\right), \quad V=\left(\bar{\lambda}^{2}, 1,-\lambda\right), \quad W=(0, \psi, 0)
$$

(which span an isomorphic lattice), so that we may take

$$
\Lambda_{3, \lambda}=J\langle U, V, W\rangle \text {. }
$$

TABLE II.

The 42 minimal vectors of $\Lambda_{3, \lambda}$ consist of these vectors and their negatives.

\begin{tabular}{|ll|ll|ll|}
\hline 10 & $(-2,-1, \lambda)$ & 20 & $(-1, \lambda,-2)$ & 40 & $(\lambda,-2,-1)$ \\
21 & $\left(1,-\bar{\lambda}, \lambda^{2}\right)$ & 31 & $\left(-\bar{\lambda}^{2},-1, \bar{\lambda}\right)$ & 51 & $\left(1,-\lambda, \lambda^{2}\right)$ \\
32 & $\left(-\lambda, \lambda^{2}, 1\right)$ & 42 & $\left(-\bar{\lambda}, \lambda^{2}, 1\right)$ & 62 & $\left(-1, \bar{\lambda},-\bar{\lambda}^{2}\right)$ \\
43 & $(\psi, 0,0)$ & 53 & $\left(\lambda,-\bar{\lambda}^{2},-1\right)$ & 03 & $(-\bar{\lambda}, 2,1)$ \\
54 & $\left(\bar{\lambda},-\bar{\lambda}^{2},-1\right)$ & 64 & $\left(\lambda^{2}, 1, \lambda\right)$ & 14 & $\left(\lambda^{2}, 1,-\bar{\lambda}\right)$ \\
65 & $\left(-1, \lambda,-\bar{\lambda}^{2}\right)$ & 05 & $(1,-\bar{\lambda}, 2)$ & 25 & $(0, \psi, 0)$ \\
06 & $(2,1, \bar{\lambda})$ & 16 & $(0,0, \psi)$ & 36 & $\left(-\vec{\lambda}^{2},-1, \lambda\right)$ \\
\hline
\end{tabular}


Then we can see that $\left(\Lambda_{3, \lambda}\right)_{\text {real }} \cong A_{6}$, for there are 42 minimal vectors in $\Lambda_{3, \lambda}$, which can be labeled with ordered pairs $i j$ with $i, j \in\{0, \ldots, 6\}, i \neq j$, as shown in Table II. These minimal vectors can also be described as all cyclic shifts of

$$
\pm(\psi, 0,0) \text { and } \pm\left(\lambda_{1} \lambda_{2}, 1,-\lambda_{3}\right)
$$

where each $\lambda_{i}$ is either $\lambda$ or $\bar{\lambda}$. The automorphism group of $\Lambda_{3, \lambda}$ has order 42 . This completes the proof of (i).

There is a certain four-dimensional $\mathbf{Z}[\lambda]$-lattice $K$, whose automorphism group is the double cover of the alternating group $\mathbf{A}_{7}$. Some years ago the first author and $R$. A. Wilson (unpublished) determined the minimal vectors in this lattice. There are 240, of norm 7, as shown in Table III. They span $K$, and show that the real form $K_{\text {real }}$ is isomorphic to $\Lambda_{8}$.

TABLE III.

The 240 minimal vectors of the lattice $K=\Lambda_{4, \lambda}$ consist of these vectors and their negatives, with each $\lambda_{i}$ replaced by either $\lambda$ or $\bar{\lambda}$.

\begin{tabular}{|l|l|l|l|}
\hline$(\psi \mid 0,0,0)$ & $(0 \mid \psi, 0,0)$ & $(0 \mid 0, \psi, 0)$ & $(0 \mid 0,0, \psi)$ \\
$\left(1 \mid \lambda_{1}, \lambda_{2}, \lambda_{3}\right)$ & $\left(-\lambda_{1} \mid 1, \lambda_{2},-\lambda_{3}\right)$ & $\left(-\lambda_{1} \mid-\lambda_{2}, 1, \lambda_{3}\right)$ & $\left(-\lambda_{1} \mid \lambda_{2},-\lambda_{3}, 1\right)$ \\
$\left(\lambda_{1} \lambda_{2} \mid-1,-1,-1\right)$ & $\left(1 \mid \lambda_{1} \lambda_{2}, 1,-1\right)$ & $\left(1 \mid-1, \lambda_{1} \lambda_{2}, 1\right)$ & $\left(1 \mid 1,-1, \lambda_{1} \lambda_{2}\right)$ \\
$\left(\lambda_{1} \lambda_{2} \mid \lambda_{3}, 1,0\right)$ & $\left(-\lambda_{1} \mid \lambda_{2} \lambda_{3}, 0,-1\right)$ & $\left(-1 \mid 0, \lambda_{1} \lambda_{2}, \lambda_{3}\right)$ & $\left(0 \mid 1,-\lambda_{1}, \lambda_{2} \lambda_{3}\right)$ \\
$\left(\lambda_{1} \lambda_{2} \mid 0, \lambda_{3}, 1\right)$ & $\left(0 \mid \lambda_{1} \lambda_{2}, 1,-\lambda_{3}\right)$ & $\left(-\lambda_{1} \mid-1, \lambda_{2} \lambda_{3}, 0\right)$ & $\left(-1 \mid \lambda_{1}, 0, \lambda_{2} \lambda_{3}\right)$ \\
$\left(\lambda_{1} \lambda_{2} \mid 1,0, \lambda_{3}\right)$ & $\left(-1 \mid \lambda_{1} \lambda_{2}, \lambda_{3}, 0\right)$ & $\left(0 \mid-\lambda_{1}, \lambda_{2} \lambda_{3}, 1\right)$ & $\left(-\lambda_{1} \mid 0,-1, \lambda_{2} \lambda_{3}\right)$ \\
\hline
\end{tabular}

Since $\Lambda_{8}$ is the densest 8-dimensional real lattice $[2,37]$, no other lattice can have a smaller determinant and the same minimal norm. Now $\Lambda_{3, \lambda}$ is a sublattice of $K$, consisting of all vectors of the form $(0 \mid \alpha, \beta, \gamma)$, and therefore $K$ is a $\Lambda_{4, \lambda}$.

The above discussion shows that $\Lambda_{8}$ has a $\lambda$-map, i.e. a fixed-point free automorphism with minimal polynomial $x^{2}+x+2$. Now $\Lambda_{16}$ can be obtained (see [8]) by gluing $\Lambda_{8}$ to a scaled copy $c \Lambda_{8}$ in such a way that minimal vectors of $c \Lambda_{8}$ glue to deep holes in $\Lambda_{8}$. The gluing map requires only the existence of a norm-doubling endomorphism of $\Lambda_{8}$. In order to equip $\Lambda_{16}$ with a $\lambda$-map we must carry out this gluing process using a norm-doubling endomorphism that commutes with a $\lambda$-map for $\Lambda_{8}$. We can use the $\lambda$-map itself for this endomorphism! Therefore $\Lambda_{16}$ has a $\lambda$-map, i.e. is a $\mathbf{Z}[\lambda]$-lattice.

Similar arguments show that we can make $\Lambda_{16}, \Lambda_{24}, \Lambda_{32}^{(a)}, \Lambda_{40}^{(a)}, \Lambda_{48}^{(a)}$ into $\mathbf{Z}[\lambda]-$ lattices, in such a way that the $\lambda$-map on $\Lambda_{8 n}$ preserves the sublattice $\Lambda_{8 n-8}$. Parts (ii)-(iv) of the theorem now follow from Theorem 3 in the usual way.

REMARK. In particular we have shown that $\Lambda_{4, i}, \Lambda_{4, \eta}, \Lambda_{4, \omega}, \Lambda_{4, \lambda}$ are different complex lattices whose real form is $\Lambda_{8}$, and $\Lambda_{12, i}, \Lambda_{12, \eta}, \Lambda_{12, \omega}, \Lambda_{12, \lambda}$ are different 
complex versions of the Leech lattice. Also, provided the real dimension does not exceed 48 , the real form of any $\Lambda_{n, \omega}$ (resp. $\Lambda_{2 n, i}, \Lambda_{2 n, \eta}$ or $\Lambda_{4 n, \lambda}$ ) is a $\Lambda_{2 n}$ (resp. $\Lambda_{4 n}, \Lambda_{4 n}$ or $\left.\Lambda_{8 n}\right)$.

\section{PART III. REAL INTEGRAL LAMINATED LATTICES}

3.1. Thompson's problem. We now turn to integral laminated lattices. Our interest in them was aroused by the following question of J. G. Thompson [36], and the remarkable nature of the answers for $M=2,3$ and 4 that has emerged from the computer results of Plesken and Pohst $[31,32]$. Theorems 8 and 9 provide a partial explanation for these results.

For a given positive number $M$, let us consider any sequence of lattices $L_{0}=\Lambda_{0}$, $L_{1}, L_{2}, \ldots$ satisfying the following conditions (for $n=0,1, \ldots$ ):

(i) $L_{n+1}$ is an $n$-dimensional integral lattice of minimal norm $M$,

(ii) $L_{n+1}$ contains $L_{n}$, and

(iii) $L_{n+1}$ has the minimal possible determinant subject to (i) and (ii).

We call such a sequence a Thompson sequence. Thompson asked whether such sequences always contain unimodular lattices (compare Theorem 1). If so, then the first unimodular lattice in the sequence is necessarily a direct summand of all later terms, and the sequence has a kind of periodicity.

For example, when $M=1$ the lattice $L_{1}=\mathbf{Z}$ is already unimodular, and in this case there is a unique Thompson sequence

$$
\Lambda_{0}, \mathbf{Z}, \mathbf{Z} \oplus \mathbf{Z}, \mathbf{Z} \oplus \mathbf{Z} \oplus \mathbf{Z}, \ldots
$$

For $M=2$ it follows from [ 2 and 37] that again the sequence is unique and the answer to Thompson's question is affirmative. For [ 2 and 37] show that $\Lambda_{0}, \ldots, \Lambda_{8}$ (see (3)) are the unique densest lattices (integral or not) in dimensions 0 to 8 . Furthermore, on the scale at which their minimal norm is 2 , these lattices are integral and nested, and $\Lambda_{8}$ is unimodular. It follows that there is a unique Thompson sequence, namely

$$
\Lambda_{0}, \Lambda_{1}, \ldots, \Lambda_{8}, \Lambda_{8} \oplus \Lambda_{1}, \ldots, \Lambda_{8} \oplus \Lambda_{8}, \Lambda_{8} \oplus \Lambda_{8} \oplus \Lambda_{1}, \ldots
$$

A similar remark partially solves the case $M=4$. For the real laminated lattices $\Lambda_{n}$ (see Figure 1) happen to be integral on the scale at which the minimal norm is 4 for all $n \leqslant 24$, and the Leech lattice $\Lambda_{24}$ is unimodular. Hence for $M=4$ the Thompson "sequence" is

$$
\Lambda_{0}, \Lambda_{1}, \ldots, \Lambda_{24}, \Lambda_{24} \oplus \Lambda_{1}, \Lambda_{24} \oplus \Lambda_{2}, \ldots, \Lambda_{24} \oplus \Lambda_{24}, \Lambda_{24} \oplus \Lambda_{24} \oplus \Lambda_{1}, \ldots
$$

However, in this case the sequence is not unique, since there are several possibilities for $\Lambda_{11}, \Lambda_{12}$ and $\Lambda_{13}$. Moreover, although both $\Lambda_{13}^{\min }$ and $\Lambda_{13}^{\max }$ can be extended to $\Lambda_{14}, \Lambda_{13}^{\text {mid }}$ cannot be extended to a lattice as dense as this. Such phenomena make it necessary to define Thompson's question more precisely. In increasing order of difficulty we might ask:

(i) For each $M$, is there a unique Thompson sequence?

(ii) For each $M$, is there a Thompson sequence that contains a unimodular lattice?

(iii) Does every Thompson sequence contain a unimodular lattice?

(iv) Does every Thompson sequence contain the same unimodular lattice? and so on. 
For the cases $M=1,2$ and 3 the answer to (iv) is yes, since $M=1$ is trivial, $M=2$ is discussed above, and Plesken and Pohst [31, 32] have handled $M=3$ by extensive computer calculations. For $M=4$ it follows from [8] that the answers to (i) and (iv) are no, and the answer to (ii) is yes. But question (iii) remains open, since lattices extending $\Lambda_{13}^{\text {mid }}$ have not been investigated (except that we know $\Lambda_{13}^{\text {mid }}$ is not part of a Thompson sequence containing $\Lambda_{24}$ ).

3.2. Integral laminated lattices. However, it is possible to make Thompson's problem more precise in a different way, by stating it as an analog of the laminated lattices problem, and it is this version that we shall adopt.

Given a positive number $M$, and with $J$ as in $\$ 2.1$, we define a family $\mathbf{L}_{n}$ of what we shall call integral laminated lattices by modifying the definition of $\$ 2.2$ slightly, changing condition (i) to read

(i) $L_{n+1}$ is integral over $J$ and has minimal norm $M$.

Of course now a different choice for $M$ will produce a completely different family of lattices, and we therefore adopt the following notation. If $J=\mathbf{Z}$, a typical lattice of $\mathbf{L}_{n}$ will be denoted by $\Lambda_{n}[M]$, if $J=\mathbf{Z}[\zeta]$ by $\Lambda_{n}^{\zeta}[M]$, if $J=\mathbf{H}_{\mathbf{z}}$ by $\Lambda_{n}^{\mathbf{H}}[M]$ (and in general by $\left.\Lambda_{n}^{J}[M]\right)$. When $J$ is complex the case $M=2$ is by far the most important, because of the connections with root systems and reflection groups, and because many interesting lattices occur as examples in this case. We therefore abbreviate $\Lambda_{n}^{\zeta}$ [2] to $\Lambda_{n}^{\zeta}$. From our earlier discussion we have

THEOREM 8. For $M=1,2$ and 4 the real integral laminated lattices $\Lambda_{n}[M]$ are given by (17), (18) and (19) respectively.

3.3. Real and integral laminated lattices with $M=3$. Consider any sequence of real integral laminated lattices with $M=3$, say $\Lambda_{0}[3], \Lambda_{1}[3], \ldots$ Let $e$ be a unit vector orthogonal to all the $\Lambda_{n}[3]$, and define new lattices

$$
T_{n+1}=\left\{x+k e: x \in \Lambda_{n}[3], k \in \mathbf{Z}, k \equiv N(x)(\bmod 2)\right\} \text {. }
$$

(The construction of $T_{n+1}$ from $\Lambda_{n}[3]$ is an analog for lattices of adding an overall parity check to a code, as in [25, p. 27].) The following properties of the $T_{n+1}$ are easily verified:

(1) $\Lambda_{n}[3]=T_{n+1} / e^{\perp}$.

(2) $T_{1}=\langle 2 e\rangle$.

(3) $T_{n+1}$ contains $T_{n}$, all its vectors have even norm, and the smallest nonzero norm is 4 .

(4) Each vector of $T_{n+1}$ has even inner product with $2 e$.

(5) $T_{n+1}$ has minimal determinant subject to (2), (3), (4).

Therefore, if there is a sequence of integral laminated lattices with $M=4$, say $\Lambda_{0}$ [4], $\Lambda_{1}[4], \ldots, \Lambda_{n+1}[4]$, with the property that

every vector of $\Lambda_{i+1}[4], 0 \leqslant i \leqslant n$, has even inner product with the generator $2 e$ (say) of $\Lambda_{1}[4]$, 
TABLE IV.

Determinants and contact numbers $\tau$ for the lattices $\Lambda_{n}$ [3] shown in Figures 5(b), (c). The entries for $n \leqslant 12$ come from Theorem 9, the remaining entries from Plesken and Pohst [31]. The contact numbers for the five 14-dimensional lattices are $\Lambda_{14}^{\min }[3]: 200, \Lambda_{14}^{\operatorname{mini}}[3]: 200, \Lambda_{14}^{\operatorname{mid}}[3]: 208, \Lambda_{14}^{\operatorname{midi}}[3]: 208, \Lambda_{14}^{\max }[3]: 224$.

\begin{tabular}{|l|l|l||l|l|l|}
\hline$n$ & $\operatorname{det} \Lambda_{n}[3]$ & $\tau$ & $n$ & $\operatorname{det} \Lambda_{n}[3]$ & $\tau$ \\
\hline \hline 0 & 1 & 0 & 12 & 256 & 136 \\
2 & 3 & 2 & 13 & 256 & 168,176 \\
3 & 8 & 4 & 14 & 256 & $200-224$ \\
4 & 16 & 8 & 15 & 192 & 264,272 \\
5 & 32 & 12 & 16 & 128 & 368,380 \\
6 & 48 & 20 & 17 & 64 & 576 \\
7 & 64 & 32 & 18 & 48 & 704 \\
8 & 128 & 60 & 20 & 16 & 1280 \\
9 & 192 & 68 & 21 & 8 & 1792 \\
10 & 256 & 80,80 & 22 & 3 & 2816 \\
11 & 256 & 104,104 & 23 & 1 & 4600 \\
\hline
\end{tabular}


then $\Lambda_{i+1}[4] / \Lambda_{1}[4]$ is a $\Lambda_{i}$ [3] sequence. In other words, we obtain a $\Lambda_{i}$ [3] sequence by projecting a $\Lambda_{i+1}$ [4] sequence satisfying (20) onto $\Lambda_{1}[4]^{\perp}$. It also follows that if (20) holds then these are the only possible $\Lambda_{i}[3]$ lattices.

We saw in Theorem 8 that for $n \leqslant 24$ the lattices $\Lambda_{n}$ [4] are the same as the laminated lattices $\Lambda_{n}$ of [8]. It is not difficult to show that the lattices displayed in Figure 5(a), but not $\Lambda_{12}^{\min }, \Lambda_{13}^{\min }, \Lambda_{13}^{\text {mid }}$ or $\Lambda_{14}$, are at the ends of sequences for which (20) holds. We have therefore established the following result.

TheOREM 9. All possibilities for $\Lambda_{0}[3], \ldots, \Lambda_{12}$ [3] are obtained by projecting the lattices of Figure 5(a) onto $\Lambda_{1}^{\perp}$, and are as shown in Figure 5(b).

There is a unique lattice $\Lambda_{n}$ [3] for $n=0, \ldots, 9$ and 12 , and precisely two inequivalent lattices each for $n=10$ and 11. Their determinants and contact numbers are shown in Table IV. The two 10-dimensional lattices $\Lambda_{10}^{\min }$ [3] and $\Lambda_{10}^{\max }$ [3] have the same contact numbers but different numbers of norm 4 vectors (352 and 358 respectively). Similarly for $\Lambda_{11}^{\min }$ [3] and $\Lambda_{11}^{\max }$ [3], which have respectively 528 and 544 norm 4 vectors.
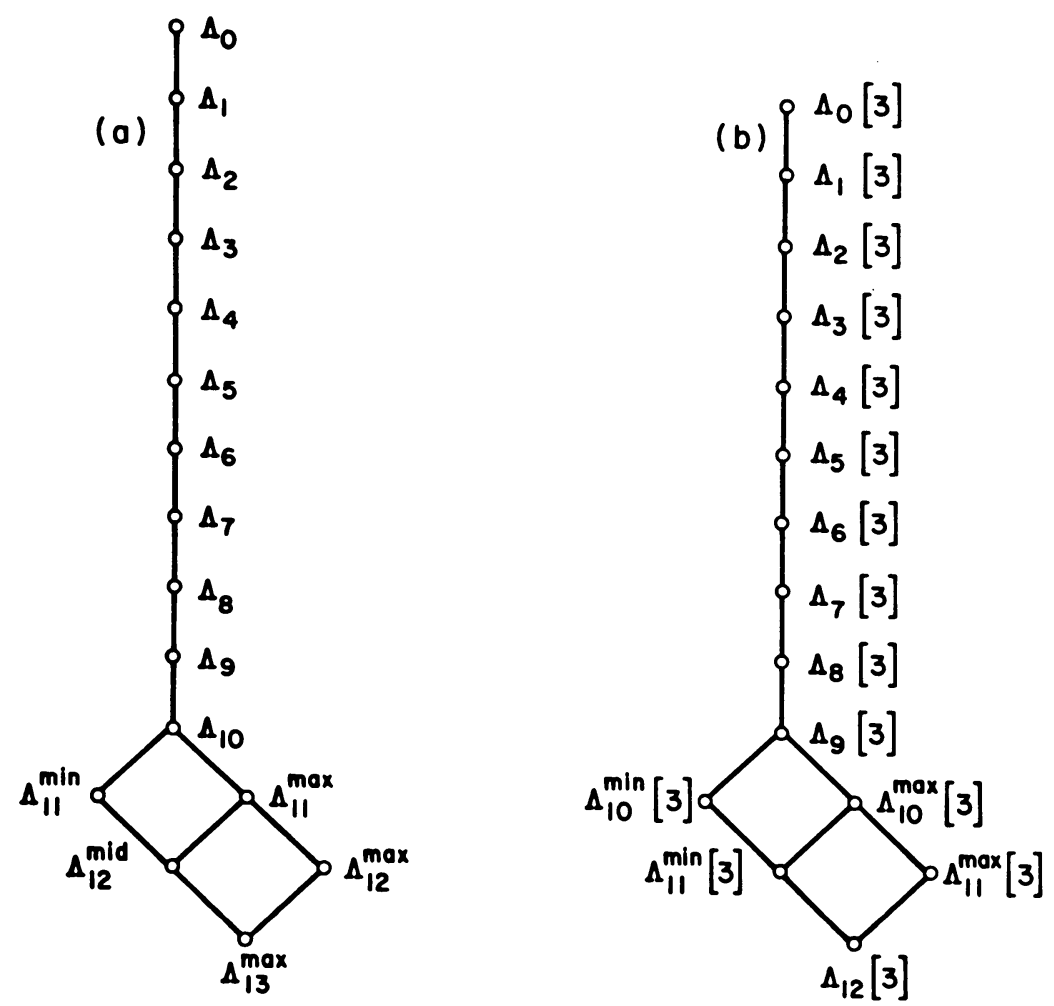

Figure 5. (a) Laminated lattices $\Lambda_{n}$ satisfying (20). (b) Projecting onto $\Lambda_{1}^{\perp}$ gives all lattices $\Lambda_{n}$ [3] for $n \leqslant 12$, see Theorem 9. These lattices were first found by Plesken and Pohst [31] using a computer. (c) All $\Lambda_{n}$ [3] for $12 \leqslant n \leqslant 23$, as found by Plesken and Pohst [31]. $\Lambda_{23}[3]$ is the shorter Leech lattice. 


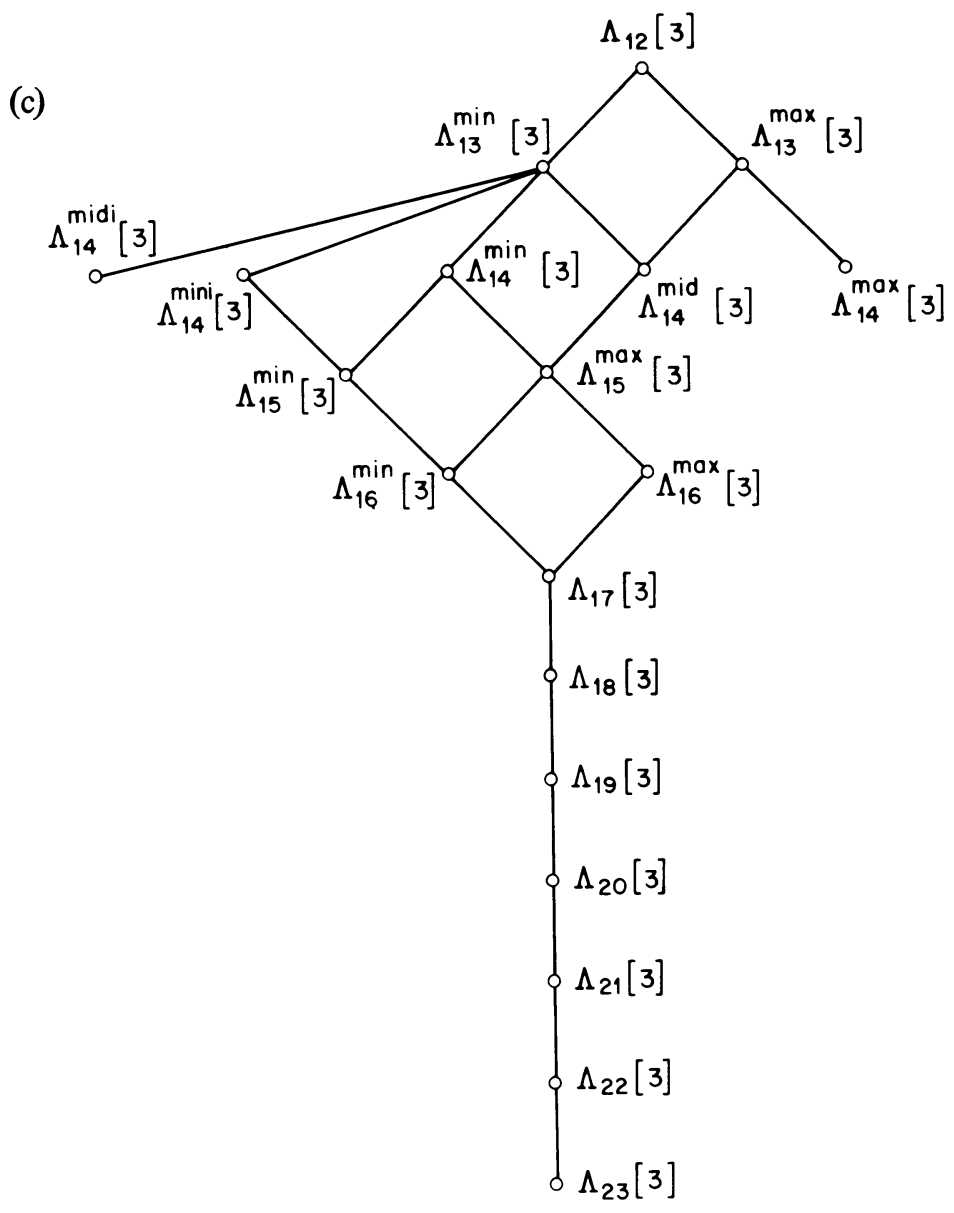

FIGURE 5. (Continued)

Theorem 9 was first established by Plesken and Pohst [31, 32], using a computer. In fact, while answering the strongest form (iv) of Thompson's question for the case $M=3$ (see §3.1), Plesken and Pohst found all the lattices $\Lambda_{n}$ [3] for $n \leqslant 23$. With their permission we include these lattices for dimensions 12 to 23 in Figure 5(c), and give the densities and contact numbers in Table IV. There is a unique $\Lambda_{23}$ [3], which is a unimodular lattice of minimal norm 3. This is the lattice sometimes called the shorter Leech lattice [9], and consists of those vectors of the Leech lattice $\Lambda_{24}$, which have even inner product with a particular minimal vector $v \in \Lambda_{24}$, projected onto $v^{\perp}$.

3.4. The best integral lattices known. In this section we investigate the densest integral lattices presently known with minimal norms $M=2,3,4, \ldots$, in dimensions $n \leqslant 24-$ see Table $\mathrm{V}$. They need not be laminated.

$M=2$. For minimal norm 2 the smallest possible determirant is known for all $n$ (see the second column of Table V). It is equal to 1 in all dimensions $n \geqslant 14$. The third column gives examples of lattices having these determinants. The lattices are 
specified by their Witt components (the indecomposable direct summands spanned by vectors of norm 2 ), with a + to indicate the presence of additional glue vectors of norm greater than 2 . For $n \leqslant 8$ see Blichfeldt [2], for $9 \leqslant n \leqslant 16$ see Kneser [20], and for larger $n$ see $[7,9]$.

\section{TABLE V.}

Smallest determinant of any integral lattice presently known with minimal norm $M=2,3$ or 4 , and examples of typical lattices having these determinants.

\begin{tabular}{|l|l|l|l|l|l|l|}
\hline \multirow{2}{*}{$n$} & \multicolumn{2}{|c|}{$M=2$} & \multicolumn{2}{|c|}{$M=3$} & \multicolumn{2}{|c|}{$M=4$} \\
\cline { 2 - 7 } & det & lattice & det & lattice & det & lattice \\
\hline \hline 0 & 1 & $\Lambda_{0}$ & 1 & $\Lambda_{0}[3]$ & 1 & $\Lambda_{0}$ \\
1 & 2 & $A_{1}$ & 3 & $\Lambda_{1}[3]$ & 4 & $\Lambda_{1}$ \\
2 & 3 & $A_{2}$ & 8 & $\Lambda_{2}[3]$ & 12 & $\Lambda_{2}$ \\
3 & 4 & $A_{3}$ & 16 & $\Lambda_{3}[3]$ & 32 & $\Lambda_{3}$ \\
4 & 4 & $D_{4}$ & 32 & $\Lambda_{4}[3]$ & 64 & $\Lambda_{4}$ \\
5 & 4 & $D_{5}$ & 48 & $\Lambda_{5}[3]$ & 128 & $\Lambda_{5}$ \\
6 & 3 & $E_{6}$ & 64 & $\Lambda_{6}[3]$ & 192 & $\Lambda_{6}$ \\
7 & 2 & $E_{7}$ & 64 & $\Lambda_{7}[3]$ & 256 & $\Lambda_{7}$ \\
8 & 1 & $E_{8}$ & 128 & $\Lambda_{8}[3]$ & 256 & $\Lambda_{8}$ \\
9 & 2 & $E_{8} A_{1}$ & 192 & $\Lambda_{9}[3]$ & 512 & $\Lambda_{9}$ \\
10 & 3 & $E_{8} A_{2}$ & 243 & $K_{10}[3]$ & 768 & $\Lambda_{10}$ \\
11 & 2 & $\left(D_{10} A_{1}\right)+$ & 256 & $\Lambda_{11}[3]$ & 972 & $K_{11}$ \\
12 & 1 & $D_{12}+$ & 256 & $\Lambda_{12}[3]$ & 729 & $K_{12}$ \\
13 & 2 & $\left(D_{6} E_{7}\right)+$ & 243 & $K_{10}[3]^{\perp}$ & 972 & $K_{13}$ \\
14 & 1 & $E_{7}^{2}+$ & 192 & $\Lambda_{9}[3]^{\perp}$ & 768 & $\Lambda_{14}$ \\
15 & 1 & $A_{15}+$ & 128 & $\Lambda_{8}[3]^{\perp}$ & 512 & $\Lambda_{15}$ \\
16 & 1 & $E_{8}^{2}$ & 64 & $\Lambda_{7}[3]^{\perp}$ & 256 & $\Lambda_{16}$ \\
17 & 1 & $\left(A_{11} E_{6}\right)+$ & 64 & $\Lambda_{6}[3]^{\perp}$ & 256 & $\Lambda_{17}$ \\
18 & 1 & $A_{9}^{2}+$ & 48 & $\Lambda_{5}[3]^{\perp}$ & 192 & $\Lambda_{18}$ \\
19 & 1 & $\left(A_{7}^{2} D_{5}\right)+$ & 32 & $\Lambda_{4}[3]^{\perp}$ & 128 & $\Lambda_{19}$ \\
20 & 1 & $E_{8} D_{12}+$ & 16 & $\Lambda_{3}[3]^{\perp}$ & 64 & $\Lambda_{20}$ \\
21 & 1 & $A_{3}^{7}+$ & 8 & $\Lambda_{2}[3]^{\perp}$ & 32 & $\Lambda_{21}$ \\
22 & 1 & $E_{8} E_{7}^{2}+$ & 3 & $\Lambda_{1}[3]^{\perp}$ & 12 & $\Lambda_{22}$ \\
23 & 1 & $E_{8} A_{15}+$ & 1 & $\Lambda_{23}[3]$ & 4 & $\Lambda_{23}$ \\
24 & 1 & $E_{8}^{3}$ & 1 & $\Delta_{24}[3]$ & 1 & $\Lambda_{24}$ \\
\hline
\end{tabular}

$M=3$. For minimal norms 3 and 4 the entries in general only give upper bounds on the smallest possible determinant. For $M=3$ we observe that since each of $\Lambda_{1}, \ldots, \Lambda_{13}^{\max }$ is contained in $\Lambda_{24}$, it follows that $\Lambda_{0}[3], \ldots, \Lambda_{12}$ [3] are contained in the shorter Leech lattice $\Lambda_{23}$ [3]. Therefore, by Theorem 4 of [8], the orthogonal lattice to 
$\Lambda_{n}$ [3] in $\Lambda_{23}$ [3] $(0 \leqslant n \leqslant 12)$, which we shall denote by $\Lambda_{n}[3]^{\perp}$, has the same determinant as $\Lambda_{n}$ [3]. It is worth noting that, although $\Lambda_{11}[3]$ and $\Lambda_{12}[3]^{\perp}$ are both 11-dimensional lattices of determinant 256 , they are not equivalent.

This has explained all the entries in the fifth column of Table V except $n=10,13$ and 24. In [8 and 21] a second sequence $K_{n}$ of sections of the Leech lattice was defined. These are integral lattices of minimal norm 4 , and for $0 \leqslant n \leqslant 10$ have the property that every vector of $K_{n+1}$ has even inner product with the generator of a suitable $K_{1}$. Therefore the lattices

$$
K_{n}[3]:=K_{n+1} / K_{1}^{\perp} \quad(0 \leqslant n \leqslant 10)
$$

are integral lattices of minimal norm 3. They are also contained in $\Lambda_{23}$ [3], and the orthogonal lattice $K_{n}[3]^{\perp}$ to $K_{n}[3]$ in $\Lambda_{23}[3]$ has the same determinant as $K_{n}$ [3]. $K_{10}[3]$ and $K_{10}[3]^{\perp}$ appear in the table.

For $n=24$ the table shows the odd Leech lattice. ${ }^{1}$ This is a 24-dimensional unimodular lattice of minimal norm 3 , which we denote by $\Delta_{24}$ [3] (although this is not part of any general notation). $\Delta_{24}[3]$ consists of the vectors $\lambda(\mathbf{0}+2 \mathbf{c}+4 \mathbf{x})$ and $\lambda(\mathbf{1}+2 \mathbf{c}+4 \mathbf{x})$, where $\lambda=2^{-3 / 2}, \mathbf{0}=(0,0, \ldots, 0), \mathbf{1}=(1,1, \ldots, 1), \mathbf{c}$ is in the binary Golay code of length 24 , and $\mathbf{x} \in \mathbf{Z}^{24}$ has the sum of its components even.

Using $\Lambda_{23}$ [3], $\Delta_{24}$ [3], $\Lambda_{32}$ and $\Lambda_{40}$ it is possible to construct unimodular lattices of minimal norm 3 in all dimensions $n \geqslant 178$. However we know from Theorem 1 that unimodular lattices of minimal norm $\geqslant 3$ exist in all dimensions $n \geqslant 37$. At present it is not known how to construct lattices.

$M=4$. For minimal norm 4 and $n \leqslant 24$, the best integral lattices known belong to either the $\Lambda_{n}$ or $K_{n}$ sequences-see the last column of Table V. From Theorem 1 unimodular lattices with minimal norm $\geqslant 4$ exist in all dimension $n \geqslant 54$.

Other values of $M$. In one dimension of course the best integral lattice for any $M$ has Gram matrix $G$ (see $\$ 2.1$ ) equal to $(M)$, of determinant $M$. In two dimensions it is easy to see using Minkowski-reduced forms that the best lattice has Gram matrix $G=\left(\begin{array}{cc}2 m-m \\ -m & 2 m\end{array}\right)$, of determinant $3 m^{2}$, if $M=2 m$, or $G=\left(\begin{array}{c}2 m+1 \\ -m\end{array} 2 m+1\right)$, of determinant $3 m^{2}+4 m+1$, if $M=2 m+1$.

In three dimensions for $M=5$ it can be shown similarly that the best lattice is

$$
G=\left(\begin{array}{rrr}
5 & -2 & -1 \\
-2 & 5 & -2 \\
-1 & -2 & 5
\end{array}\right)
$$

of determinant 72 . For $M=6$ the best is

$$
G=\left(\begin{array}{rrr}
6 & -3 & 0 \\
-3 & 6 & -3 \\
0 & -3 & 6
\end{array}\right)
$$

of determinant 108 , obtained by scaling the best matrix for minimum 2 . Similarly for any even $M=2 m$ the best lattices in dimensions $n=0,1, \ldots, 8$ are obtained by rescaling $\Lambda_{n}$ [2], and have determinants

$$
1,2 m, 3 m^{2}, 4 m^{3}, 4 m^{4}, 4 m^{5}, 3 m^{6}, 2 m^{7}, m^{8}
$$

respectively.

1 Added in proof. Apparently first discovered by O'Connor and Pall, Duke Math. J. 11 (1944), 319-331. 


\section{PART IV. COMPLEX AND QUATERNIONIC INTEGRAL LAMINATED LATTICES}

4.1. Method. The real, complex and quaternionic integral laminated lattices of minimal norm $2, \Lambda_{n}^{J}[2]$ (which we abbreviate to $\Lambda_{n}^{\zeta}$ when $J=\mathbf{Z}[\zeta]$ ), may be found by a uniform method, applicable to all seven of our rings $J$. Of course we have already dealt with the case $J=\mathbf{Z}$ in Theorem 8 . However this argument will enable us to obtain the result stated there (for $M=2$ ) without appealing to the work of Blichfeldt and Vetchinkin. In this section we sketch the method, and then in $\$ 4.2$ we give a detailed account of the most difficult case, when $J=\mathbf{Z}[\omega]$. The other cases are dealt with in the following sections.

\section{TABLE VI.}

The lowest-dimensional unimodular lattices $\Lambda_{N}^{J}$ [2] and their minimal vectors. The last column gives the number of minimal vectors of each type. The coordinates can usually be permuted, and there may be restrictions on them. (If in doubt compare the last two columns and consult the text.)

\begin{tabular}{|c|c|c|c|c|}
\hline$J$ & $N$ & $\Lambda_{N}^{J}[2]$ & minimal vectors & number \\
\hline $\mathbf{Z}$ & 8 & $\begin{array}{r}\Lambda_{8}[2]=\Lambda_{8} \\
\left(=E_{8}\right)\end{array}$ & $\begin{array}{l}\left( \pm 1^{2}, 0^{6}\right)_{1} \\
\left( \pm \frac{1}{2}^{8}\right)_{1}\end{array}$ & $\begin{array}{c}2^{2}\left(\begin{array}{l}8 \\
2\end{array}\right) \\
2^{7}\end{array}$ \\
\hline $\mathbf{Z}[\omega]$ & 6 & $\Lambda_{6}^{\omega}$ & $\begin{array}{l} \pm\left(\omega^{a}, \ldots, \omega^{f}\right)_{3} \\
\pm\left(\omega^{a} \theta,-\omega^{b} \theta, 0^{4}\right)_{3}\end{array}$ & $\begin{array}{c}2 \cdot 3^{5} \\
2 \cdot 3^{2}\left(\begin{array}{c}6 \\
2\end{array}\right)\end{array}$ \\
\hline $\mathbf{Z}[i]$ & 4 & $\begin{aligned} & \Lambda_{4}^{i}=\Lambda_{4, i} \\
&\left(\cong E_{8}\right)\end{aligned}$ & $\begin{array}{l}\left(i^{a}, \ldots, i^{d}\right)_{2} \\
\left(2 i^{a}, 0^{3}\right)_{2} \\
( \pm 1 \pm i, \pm 1 \pm i, 0,0)_{2}\end{array}$ & $\begin{array}{c}2^{7} \\
4^{2} \\
2^{4}\left(\frac{6}{2}\right)\end{array}$ \\
\hline $\mathbf{Z}[\lambda]$ & 3 & $\Lambda_{3}^{\lambda}$ & $\begin{array}{l}( \pm \lambda, \pm 1, \pm 1)_{2} \\
( \pm \bar{\lambda}, \pm \bar{\lambda}, 0)_{2} \\
( \pm 2,0,0)_{2}\end{array}$ & $\begin{array}{l}2^{3} \cdot 3 \\
2^{2} \cdot 3 \\
2 \cdot 3\end{array}$ \\
\hline $\mathbf{Z}[\eta]$ & 2 & $\begin{array}{c}\Lambda_{2}^{\eta}=\Lambda_{2, \eta} \\
\left(\cong D_{4}\right)\end{array}$ & $\begin{array}{l}( \pm 2,0)_{2} \\
( \pm \eta, \pm \eta)_{2} \\
( \pm \eta \pm 1, \pm 1)_{2}\end{array}$ & $\begin{array}{l}2^{2} \\
2^{2} \\
2^{4}\end{array}$ \\
\hline $\mathbf{Z}[\nu]$ & 2 & $\Lambda_{2}^{\prime}$ & $\begin{array}{l}( \pm 2,0)_{2} \\
( \pm \nu, \pm 1)_{2}\end{array}$ & $\begin{array}{l}2^{2} \\
2^{3}\end{array}$ \\
\hline $\mathbf{H}_{\mathbf{z}}$ & 2 & $\begin{aligned} & \Lambda_{2}^{H}[2]=\Lambda_{2, H} \\
&\left(\cong E_{8}\right)\end{aligned}$ & $\begin{array}{l}( \pm 1 \pm j, 0)_{1} \\
( \pm 1, \pm j)_{1} \\
\left(\omega^{j}, \omega^{k}\right)_{1}\end{array}$ & $\begin{array}{c}2^{3}\left(\begin{array}{l}4 \\
2\end{array}\right) \\
2^{6} \\
2^{7}\end{array}$ \\
\hline
\end{tabular}


Step 1. For each of the seven rings $J$ it turns out that there is a low-dimensional lattice $\Lambda_{N}^{J}$ [2] which is unimodular. These seven lattices together with their minimal vectors are shown in Table VI (and defined in more detail below). In describing these lattices we use the subscript notation introduced in [10], according to which $\left(x_{1}, \ldots, x_{n}\right)_{c}$ stands for $z^{-1}\left(x_{1}, \ldots, x_{n}\right)$, where $z$ is an appropriate constant of norm $c$. In particular the norm of $\left(x_{1}, \ldots, x_{n}\right)_{c}$ is $\Sigma\left|x_{i}\right|^{2} / c$. Since we have not yet proved that the lattices shown in Table VI are $\Lambda_{N}^{J}$ [2]'s, we shall temporarily denote a typical lattice in the table by $L_{N}$.

Step 2. Inside each $L_{N}$ there is a chain of sections

$$
L_{0} \subseteq L_{1} \subseteq \cdots \subseteq L_{N},
$$

defined in Table VII (these will turn out later to be the lattices $\left.\Lambda_{n}^{J}[2]\right)$. In this table a small circle represents a zero coordinate, a hollow loop is a set of coordinates adding to zero, an asterisk is a free coordinate, and a line of asterisks is a set of equal coordinates. For example the entry for $n=3$ in the $\mathbf{Z}[\omega]$ column indicates that $L_{3}$ ( $=\Lambda_{3}^{\omega}$ ) consists of all vectors of $L_{6}\left(=\Lambda_{6}^{\omega}\right)$ in which the first three coordinates add to zero and the last three are equal.

\section{TABLE VII.}

The lattices $\Lambda_{n}^{J}$ [2] for $0 \leqslant n \leqslant N$, defined as sections of the $\Lambda_{N}^{J}$ [2] shown in Table VI. The notation is explained in the text. The second line of the entry for each lattice $\Lambda_{n}^{J}$ [2] gives the index of the lattice in its dual (which is $\operatorname{det} \Lambda_{n}^{J}[2]$ if $J=\mathbf{Z}, \operatorname{det}^{2} \Lambda_{n}^{J}$ [2] if $J$ is complex, or $\operatorname{det}^{4} \Lambda_{n}^{J}$ [2] if $J=\mathbf{H}_{\mathbf{z}}$ ), and also its contact number.

\begin{tabular}{|c|c|c|c|c|c|c|c|c|c|}
\hline$n$ & & & & $\omega]$ & $z[i]$ & $z[\lambda]$ & $z[\eta]$ & $z[\nu]$ & $\mathrm{Hz}_{2}$ \\
\hline \multirow{2}{*}{0} & \multicolumn{2}{|c|}{1000000001} & \multicolumn{2}{|c|}{10000001} & $10000)$ & 10001 & 1001 & 1001 & $(00)$ \\
\hline & 1 & 0 & 1 & 0 & 10 & 10 & 10 & 10 & 10 \\
\hline \multirow{2}{*}{ I } & \multicolumn{2}{|c|}{100000001} & \multicolumn{2}{|c|}{1000001} & $(* 000)$ & $(* 00)$ & $1 * 01$ & $(* 0)$ & $(* 0)$ \\
\hline & 2 & 2 & 4 & 6 & 44 & 42 & 42 & 42 & 1624 \\
\hline \multirow{2}{*}{2} & \multicolumn{2}{|c|}{10000001} & \multicolumn{2}{|c|}{100001} & $(* * 00)$ & $(* *-k)$ & $(* *)$ & (**) & (**) \\
\hline & 3 & 6 & 9 & 18 & 424 & 48 & 124 & 112 & 1240 \\
\hline \multirow{2}{*}{3} & \multicolumn{2}{|c|}{$(* * * 000000)$} & \multicolumn{2}{|c|}{ (C)***) } & $(* * * 0)$ & (***) & & & \\
\hline & 4 & 12 & 9 & 54 & 460 & 142 & & & \\
\hline \multirow{2}{*}{4} & \multicolumn{2}{|c|}{$(* * * * 0000)$} & \multicolumn{2}{|c|}{ (*******) } & $(* * * *)$ & & & & \\
\hline & 4 & 24 & 9 & 108 & 1240 & & & & \\
\hline \multirow{2}{*}{5} & \multicolumn{2}{|c|}{$(* * * * * 0001$} & \multicolumn{2}{|c|}{ (*******) } & & & & & \\
\hline & 4 & 40 & & 270 & & & & & \\
\hline \multirow{2}{*}{6} & \multicolumn{2}{|c|}{$(* * * * * * * * *)$} & \multicolumn{2}{|c|}{ (*******)} & & & & & \\
\hline & 3 & 72 & & 756 & & & & & \\
\hline \multirow{2}{*}{7} & \multicolumn{2}{|c|}{ (**********)} & & & & & & & \\
\hline & 2 & 126 & & & & & & & \\
\hline \multirow{2}{*}{8} & \multicolumn{2}{|c|}{ 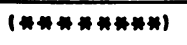 } & & & & & & & \\
\hline & 1 & 240 & & & & & & & \\
\hline
\end{tabular}

Step 3. For $0 \leqslant n \leqslant N$ let $L_{n}^{\perp}$ denote the orthogonal complement of $L_{n}$ in $L_{N}$. In each case we can verify that $L_{n}^{\perp} \cong L_{N-n}$, and so we define $L_{N-n}^{\prime}=L_{n}^{\perp}$. Then

$$
L_{n}^{*} / L_{n} \cong L_{N-n}^{\prime *} / L_{N-n}^{\prime},
$$

and $L_{N}$ is formed by gluing $L_{n}$ to $L_{N-n}^{\prime}$. In every case this gluing is carried out minimally, in the sense that, when a congruence class of $L_{n}^{*} / L_{n}$ of norm $a$ is glued to 
a class of $L_{N-n}^{\prime *} / L_{N-n}^{\prime}$ of norm $b$, we have $a+b=2$, and not merely $a+b \geqslant 2$. (The norm of a set is the smallest norm of any of its elements.)

Step 4. Aut $\left(L_{n}\right)$ is transitive on congruence classes of $L_{n}^{*} / L_{n}$ of greatest norm. Usually this transitivity is trivial, being accomplished by multiplication by units. The $\mathbf{Z}[\omega]$-lattice $L_{3}$ is the only complicated case.

Step 5. It then follows by induction that, for $0 \leqslant n \leqslant N, \Lambda_{n}^{J}[2]$ is unique and is equal to $L_{n}$. Thus by the end of Part IV we will have established

THEOREM 10. If $J$ is any of our seven rings, there is a unique sequence $\Lambda_{n}^{J}$ [2] given by

$$
L_{0}, L_{1}, \ldots, L_{N}, L_{N} \oplus L_{1}, \ldots, L_{N} \oplus L_{N}, L_{N} \oplus L_{N} \oplus L_{1}, \ldots,
$$

where the lattices $L_{n}$ are defined in Tables VI and VII.

4.2. The lattices $\Lambda_{n}^{\omega}$. The Coxeter-Todd lattice. When $J=\mathbf{Z}[\omega]$, for the lattice $L_{6}$ mentioned in Step 1 we take the six-dimensional lattice constructed by Coxeter and Todd in [15], the real form of which is $K_{12}$ (see also [8, 21, 34 and especially 10]). We shall see that its correct name is $\Lambda_{6}^{\omega}$. This lattice may be defined as the set of all vectors $\left(x_{1}, \ldots, x_{6}\right)_{3} \in \mathbf{Z}[\omega]^{6}$ satisfying $x_{1} \equiv \cdots \equiv x_{6}(\bmod \theta:=\sqrt{-3})$ and $\Sigma x_{i} \equiv 0$ (mod 3). Its automorphism group is Mitchell's complex reflection group of order 108.9! (No. 34 on Shephard and Todd's list), isomorphic to $6 \cdot U_{4}(3) \cdot 2$ (see $[3,10$, 27, 33]).

The sections $L_{0} \subseteq L_{1} \subseteq \cdots \subseteq L_{6}$ mentioned in Step 2 are defined in Table VII. From this table we see that $L_{1}$ is $\mathbf{Z}[\omega]\left\langle(-\theta, \theta, 0,0,0,0)_{3}\right\rangle$, and thus $\Lambda_{1}^{\omega}=L_{1}$.

$\Lambda_{2}^{\omega}$. To find $\Lambda_{2}^{\omega}$ it is necessary to determine the congruence classes of $L_{1}^{*} / L_{1}$ of maximal norm. This may be done using the fact (discussed in Step 3) that $L_{6}$ is formed by gluing $L_{1}$ to $L_{1}^{\perp}=L_{5}^{\prime}$. From Table VII we see that $L_{2}$ is obtained from $L_{1}$ by adjoining the vector $v=(0,-\theta, \theta, 0,0,0)_{3}$, and we may decompose

$$
v=\left(\frac{\theta}{2}, \frac{-\theta}{2}, 0,0,0,0\right)_{3}+\left(\frac{-\theta}{2}, \frac{-\theta}{2}, \theta, 0,0,0\right)_{3}=h+t \quad \text { (say), }
$$

where $h \in \mathbf{R} L_{1}, t \in \mathbf{R} L_{1}^{\perp}, N(h)=\frac{1}{2}, N(t)=\frac{3}{2}$. As representatives for the classes of $\Lambda_{1}^{*} / \Lambda_{1}$ we take $\{0, h, \omega h, \bar{\omega} h\}$, and for the classes of $\Lambda_{5}^{\prime *} / \Lambda_{5}^{\prime}$ we take $\{0, t, \omega t, \bar{\omega} t\}$. The norms of these eight classes are at most $\left\{0, \frac{1}{2}, \frac{1}{2}, \frac{1}{2}\right\}$ and $\left\{0, \frac{3}{2}, \frac{3}{2}, \frac{3}{2}\right\}$, respectively, since these are the norms of the representatives. But $L_{6}$ has minimal norm 2 , and is formed by gluing these classes together. Therefore these numbers are in fact the norms of the eight classes. This is why it is important that $\Lambda_{1}$ is glued minimally to $\Lambda_{5}^{\prime}$, as described in Step 3.

Step 4. It is clear that $\operatorname{Aut}\left(L_{1}\right)$ is transitive on the congruence classes of $L_{1}^{*} / L_{1}$ of greatest norm (for multiplication by $\omega$ permutes the nonzero classes). We conclude that $\Lambda_{2}^{\omega}$ is unique, and $\Lambda_{2}^{\omega}=L_{2}$.

An equivalent definition is $\Lambda_{2}^{\omega}=\mathbf{Z}[\omega]\langle x, y\rangle$, where $x=(2,0)_{2}, y=(-1, \sqrt{3})_{2}$. Then the minimal vectors are $\pm \omega^{i} x, \pm \omega^{i} y, \pm \omega^{i}(x+y)$, and now it is easy to see that

$$
\Lambda_{2}^{\omega}=\mathbf{Z}[\omega] A_{2} .
$$

$\Lambda_{3}^{\omega}$. We next find the classes of $L_{2}^{*} / L_{2}$, a group of order 9 (the orders of $L_{n}^{*} / L_{n}$ are given in Table VII). $L_{3}$ is obtained by adjoining

$$
(1, \omega, \bar{\omega}, 1,1,1)_{3}=(1, \omega, \bar{\omega}, 0,0,0)_{3}+(0,0,0,1,1,1)_{3}=h+t
$$


to $L_{2}$, where $h \in \mathbf{R} L_{2}, t \in \mathbf{R} L_{2}^{\perp}, N(h)=N(t)=1$. As representatives for the classes of $L_{2}^{*} / L_{2}$ we take $\left\{0, \pm \omega^{i} u, \pm \theta u\right\}$, where $u=(\theta / 3, \theta / 3,-2 \theta / 3,0,0,0)_{3}$, of norms at most $\left\{0, \frac{2}{3}, \frac{2}{3}, \frac{2}{3}, \frac{2}{3}, \frac{2}{3}, \frac{2}{3}, 1,1\right\}$ respectively. The class $\theta u$ contains $h$. As representatives for the classes of $L_{4}^{\prime *} / L_{4}^{\prime}$ we take $\left\{0, \pm \omega^{i} x, \pm \theta x\right\}$, where $x=$ $(-\theta / 3,-\theta / 3,-\theta / 3,0,0, \theta)_{3}$, of norms at most $\left\{0, \frac{4}{3}, \frac{4}{3}, \frac{4}{3}, \frac{4}{3}, \frac{4}{3}, \frac{4}{3}, 1,1\right\}$ respectively. The class $\theta x$ contains $t$. Then we see that $\Lambda_{2}$ is glued minimally to $\Lambda_{4}^{\prime}$, and so these norms are in fact the norms of the 18 classes. The argument used before now shows that $\Lambda_{3}^{\omega}$ is unique and $\Lambda_{3}^{\omega}=L_{3}$.

The minimal vectors of $\Lambda_{3}^{\omega}=L_{3}$ are listed and named in Table VIII. Given two projectively distinct minimal vectors $\alpha, \beta$ there is a projectively unique third minimal vector $\gamma$ such that

$$
\alpha+\omega^{i} \beta+\omega^{j} \gamma=0
$$

for suitable $i, j$. Such a triple $\{\alpha, \beta, \gamma\}$ we call consistent. $\left\{c_{02}, c_{24}, c_{40}\right\}$ is an example.

Three independent minimal vectors $\alpha, \beta, \gamma$ form an inconsistent triple. After multiplying by powers of $\pm \omega$ and relabeling, any inconsistent triple can be put into the form $\{\alpha, \beta, \gamma\}$ with

$$
\alpha \cdot \bar{\beta}=\beta \cdot \bar{\gamma}=-1, \quad \gamma \cdot \bar{\alpha}=-\omega .
$$

$\left\{c_{24}, b_{4}, c_{45}\right\}$ is an example. Thus all inconsistent triples are equivalent under $\operatorname{Aut}\left(\Lambda_{3}^{\omega}\right)$.

TABLE VIII.

The 54 minimal vectors of $\Lambda_{3}^{\omega}$ consist of these vectors and their negatives.

\begin{tabular}{|c|c|c|c|}
\hline \multirow{2}{*}{$\begin{array}{c}\text { vectors } \\
(i=0,1,2)\end{array}$} & \multicolumn{3}{|c|}{ names } \\
\cline { 2 - 4 } & $i=0$ & $i=1$ & $i=2$ \\
\hline$\omega^{i}(1, \omega, \bar{\omega}, 1,1,1)_{3}$ & $c_{24}$ & $a_{4}$ & $b_{2}$ \\
$\omega^{i}(\bar{\omega}, 1, \omega, 1,1,1)_{3}$ & $c_{40}$ & $a_{0}$ & $b_{4}$ \\
$\omega^{i}(\omega, \bar{\omega}, 1,1,1,1)_{3}$ & $c_{02}$ & $a_{2}$ & $b_{0}$ \\
$-\omega^{i}(1, \bar{\omega}, \omega, 1,1,1)_{3}$ & $c_{51}$ & $b_{5}$ & $a_{1}$ \\
$-\omega^{i}(\omega, 1, \bar{\omega}, 1,1,1)_{3}$ & $c_{13}$ & $b_{1}$ & $a_{3}$ \\
$-\omega^{i}(\bar{\omega}, \omega, 1,1,1,1)_{3}$ & $c_{35}$ & $b_{3}$ & $a_{5}$ \\
$\omega^{i}(0,-\theta, \theta, 0,0,0)_{3}$ & $c_{03}$ & $c_{45}$ & $c_{21}$ \\
$\omega^{i}(\theta, 0,-\theta, 0,0,0)_{3}$ & $c_{25}$ & $c_{01}$ & $c_{43}$ \\
$\omega^{i}(-\theta, \theta, 0,0,0,0)_{3}$ & $c_{41}$ & $c_{23}$ & $c_{05}$ \\
\hline
\end{tabular}


The number of projectively distinct inconsistent triples is $9 \cdot 8 \cdot 6 / 6=72$, and the subgroup of $\operatorname{Aut}\left(\Lambda_{3}^{\omega}\right)$ fixing an inconsistent triple projectively has order 18 (3 cyclic shifts, 3 multiplications by $\omega, 2$ sign changes). Therefore

$$
\left|\operatorname{Aut}\left(\Lambda_{3}^{\omega}\right)\right|=72 \cdot 18=2^{4} \cdot 3^{4} \text {. }
$$

Our names for the minimal vectors make it easy to identify the real form of $\Lambda_{3}^{\omega}$. We recall (see for example $[7,11,12]$ ) that the lattice $E_{6}$ may be defined as the set all vectors $\left(x_{0}, \ldots, x_{7}\right)$ of $E_{8}$ for which $x_{0}+\cdots+x_{5}=x_{6}+x_{7}=0$. Then the minimal vectors of $E_{6}^{*}$ consist of $\pm a_{i}, \pm b_{j}, \pm c_{i j}(0 \leqslant i, j \leqslant 5)$, where typical vectors are

$$
a_{2}=\left(-\frac{1}{6}, \frac{5}{6},-\frac{1}{6},-\frac{1}{6},-\frac{1}{6},-\frac{1}{6} \mid \frac{1}{2},-\frac{1}{2}\right)
$$

$\left(a_{i}\right.$ has the $\frac{5}{6}$ in position $i$ ),

$$
b_{3}=\left(-\frac{1}{6},-\frac{1}{6}, \frac{5}{6},-\frac{1}{6},-\frac{1}{6},-\frac{1}{6} \mid-\frac{1}{2}, \frac{1}{2}\right)
$$

$\left(b_{j}\right.$ has the $\frac{5}{6}$ in position $j$ ), and

$$
c_{12}=\left(-\frac{2}{3},-\frac{2}{3}, \frac{1}{3}, \frac{1}{3}, \frac{1}{3}, \frac{1}{3} \mid 0,0\right),
$$

( $c_{i j}$ has the $-\frac{2}{3}$ 's in positions $i, j$ ). It follows that

$$
\left(\Lambda_{3}^{\omega}\right)_{\text {real }} \cong E_{6}^{*} \text {. }
$$

$\Lambda_{4}^{\omega} . L_{4}$ is obtained by adjoining

$$
(1,1,1,1,1,1)_{3}=(0,0,0,1,1,1)_{3}+(1,1,1,0,0,0)_{3}=h+t \quad \text { (say) }
$$

to $L_{3}$, where $h \in \mathbf{R} L_{3}, t \in \mathbf{R} L_{3}^{\perp}, N(h)=N(t)=1$. Also $h=\theta^{-1}\left(a_{4}-b_{4}\right)$. It can now be shown that the 8 nontrivial classes of $L_{3}^{*} / L_{3}$ all have representatives (such as $h$ ) of the form $\theta^{-1}(\alpha-\beta)$, where $\alpha \cdot \bar{\beta}=-\omega$ or $-\bar{\omega}$, and that $\operatorname{Aut}\left(L_{3}\right)$ acts transitively on these classes. $L_{3}$ is glued minimally to $L_{3}^{\prime}$, so all classes have norm 1 . It follows as usual that $\Lambda_{4}^{\omega}$ is unique and $\Lambda_{4}^{\omega}=L_{4}$.

$\Lambda_{5}^{\omega}$ and $\Lambda_{6}^{\omega}$. We have already found the classes of $L_{4}^{\prime *} / L_{4}^{\prime}$ and $L_{5}^{\prime *} / L_{5}^{\prime}$, so the remaining cases $\Lambda_{5}^{\omega}$ and $\Lambda_{6}^{\omega}$ present no further difficulty. This completes the proof of Theorem 10 in the case $J=\mathbf{Z}[\omega]$.

4.3. The lattices $\Lambda_{n}^{i}$. The proofs for the other cases of Theorem 10 are simpler and our treatment will be brief. For $L_{4}=\Lambda_{4}^{i}$ we use the lattice $\Lambda_{4, i}$ described in $\$ 2.7$. This is a complex version of $E_{8}$, with minimal vectors of the form

$$
\begin{gathered}
\left(i^{a}, i^{b}, i^{c}, i^{d}\right)_{2}, \quad a+b+c+d \text { even, } \\
\left(2 i^{a}, 0,0,0\right)_{2}, \\
( \pm 1 \pm i, \pm 1 \pm i, 0,0)_{2},
\end{gathered}
$$

where $a, b, c, d \in\{0,1,2,3\}$ (see Table VI). The sections are

$$
\begin{aligned}
& L_{1} \cong \mathbf{Z}[i]\left\langle(2)_{2}\right\rangle, \\
& L_{2} \cong \mathbf{Z}[i]\left\langle(2,0)_{2},(1+i, 1+i)_{2}\right\rangle, \\
& L_{3} \cong \mathbf{Z}[i]\left\langle(2,0,0)_{2},(1+i, 1+i, 0)_{2},(1+i, 0,1+i)_{2}\right\rangle .
\end{aligned}
$$


Representatives for the classes of $L_{1}^{*} / L_{1}$ are $\left\{0,(1)_{2},(i)_{2},(1+i)_{2}\right\}$, of norms $\left\{0, \frac{1}{2}, \frac{1}{2}, 1\right\}$, and these are glued to the classes $\left\{0,(1,1,1)_{2},(i, i, i)_{2},(1+i, 0,0)_{2}\right\}$ of $L_{3}^{\prime *} / L_{3}^{\prime}$, of norms $\left\{0, \frac{3}{2}, \frac{3}{2}, 1\right\}$. The classes of $L_{2}^{*} / L_{2}$ are $\left\{0,(1,1)_{2},(1, i)_{2},(1+i, 0)_{2}\right\}$, of norms $\{0,1,1,1\}$, and are glued to copies of themselves.

4.4. The lattices $\Lambda_{n}^{\lambda}$. When $J=Z[\lambda]$ we take

$$
\begin{aligned}
& L_{1}=J\left\langle(2,0,0)_{2}\right\rangle, \\
& L_{2}=J\left\langle(2,0,0)_{2},(\lambda, 1,1)_{2}\right\rangle, \\
& L_{3}=J\left\langle(2,0,0)_{2},(\lambda, 1,1)_{2},(\bar{\lambda}, \bar{\lambda}, 0)_{2}\right\rangle .
\end{aligned}
$$

$\operatorname{Aut}\left(L_{3}\right)$ is Klein's complex reflection group of order 336 (No. 24 on Shephard and Todd's list), and is isomorphic to $2 \times L_{2}(7)$ (see [3, 33]).

We find that $L_{1}^{*}=\frac{1}{2} L_{1}$, and as representatives for the classes of $L_{1}^{*} / L_{1}$ we may take $\left\{0,(1,0,0)_{2},(\lambda, 0,0)_{2},(\bar{\lambda}, 0,0)_{2}\right\}$, of norms $\left\{0, \frac{1}{2}, 1,1\right\}$ respectively. These are glued minimally to the classes $\left\{0,(0, \lambda, 1)_{2},(0,1,1)_{2},(0, \bar{\lambda}, 0)_{2}\right\}$ of $L_{2}^{\prime *} / L_{2}^{\prime}$, of norms $\left\{0, \frac{3}{2}, 1,1\right\}$. With one exception, which concerns the uniqueness of $\Lambda_{2}^{\lambda}$, the argument continues as before. $\Lambda_{2}^{\lambda}$ is obtained by adjoining either $(\lambda, 1,1)_{2}$ or $(\bar{\lambda}, 1,1)_{2}$ to $\Lambda_{1}^{\lambda}=L_{1}=\mathbf{Z}[\lambda]\left\langle(2,0,0)_{2}\right\rangle$. Now there is an element of $S U(3, \mathbf{C})$ which sends $\mathbf{Z}[\lambda]\left\langle(2,0,0)_{2},(\lambda, 1,1)_{2}\right\rangle$ into $\mathbf{Z}[\lambda]\left\langle(2,0,0)_{2},(\bar{\lambda}, 1,1)_{2}\right\rangle$, but it is not possible to do this while preserving $\Lambda_{1}^{\lambda}$. Thus although $\Lambda_{2}^{\lambda}$ is unique, the pair $\left(\Lambda_{1}^{\lambda}, \Lambda_{2}^{\lambda}\right)$ is not.

4.5. The remaining cases. In the case $J=\mathbf{Z}[\eta]$ we take $L_{2}$ to be the lattice $\Lambda_{2, \eta}$ described in $\$ 2.7$ (a complex version of $\Lambda_{4}$ ). It is now easy to complete the argument using the information in Tables VI and VII. For $J=\mathbf{Z}[\nu]$ the argument is even simpler. In the real case, when $J=\mathbf{Z}$, the argument is longer, but all necessary information about the congruence classes of $\Lambda_{n}^{*} / \Lambda_{n}$ is available in [8].

For $J=\mathbf{H}_{\mathbf{z}}$ we take $L_{2}=\Lambda_{2, \mathbf{H}}$ (see $\left.\$ 2.6\right)$, a quaternionic version of $E_{8}$. Again the argument is straightforward. This completes the proof of Theorem 10.

Acknowledgement. We are very grateful to W. Plesken and M. Pohst for allowing us to mention some of their results on Thompson's problem.

\section{REFERENCES}

1. A. Baker, Linear forms in the logarithms of algebraic numbers. I, Mathematika 13 (1966), 204-216.

2. H. F. Blichfeldt, The minimum values of positive quadratic forms in six, seven and eight variables, Math. Z. 39 (1934), 1-15.

3. A. M. Cohen, Finite complex reflection groups, Ann. Sci. École Norm. Sup. 9 (1976), 379-436.

i. I. H. Conway, Three lectures on exceptional groups in Finite Simple Groups (M. B. Powell and G. Higman, eds.), Academic Press, New York, 1971, pp. 215-247.

5. ed.), Academic Press, New York, 1977, pp. 215-247.

6. J. H. Conway, R. A. Parker and N. J. A. Sloane, The covering radius of the Leech lattice, Proc. Roy. Soc. London Ser. A 380 (1982), 261-290.

7. J. H. Conway and N. J. A. Sloane, On the enumeration of lattices of determinant one, J. Number Theory 15 (1982), 83-94.

8. ___ Laminated lattices, Ann. of Math. 116 (1982), 593-620.

9. The unimodular lattices of dimension up to 23 and the Minkowski-Siegel mass constants, European J. Combin. 3 (1982), 219-231.

10. The Coxeter-Todd lattice, the Mitchell group, and related sphere packings, Math. Proc. Cambridge Philos. Soc. 93(1983), 421-440. 
11. H. S. M. Coxeter, The polytope $2_{21}$, whose twenty-seven vertices correspond to the lines on the general cubic surface, Amer. J. Math. 62 (1940), 457-486.

12. __ Extreme forms, Canad. J. Math. 3 (1951), 391-441.

13.

14. H. S. M. Coxeter and W. O. J. Moser, Generators and relations for discrete groups, 4th ed., Springer-Verlag, New York, 1980.

15. H. S. M. Coxeter and J. A. Todd, An extreme duodenary form, Canad. J. Math. 5 (1953), 384-392.

16. R. T. Curtis, On subgroups of · 0. 1. Lattice stabilizers, J. Algebra 27 (1973), 549-573.

17. A new combinatorial approach to $M_{24}$, Math. Proc. Cambridge Philos. Soc. 79 (1976), $25-42$.

18. G. H. Hardy and E. M. Wright, An introduction to the theory of numbers, 5 th ed., Oxford Univ. Press, Oxford, 1979.

19. A. Hurwitz, Uber die Zahlentheorie der Quaternionen, Nachr. Gesellschaft Wiss. Göttingen Math.-

Phys. K1. (1896), 313-340. Reprinted in Math. Werke. Vol. II, Birkhäuser, Basel, 1933, pp. 303-330.

20. M. Kneser, Kiassenzahlen definiter quadratischer Formen, Arch. Math. 8 (1957), 241-250.

21. J. Leech and N. J. A. Sloane, Sphere packing and error-correcting codes, Canad. J. Math. 23 (1971), $718-745$.

22. J. H. Lindsey II, A correlation between $\mathrm{PSU}_{4}(3)$, the Suzuki group, and the Conway group, Trans. Amer. Math. Soc. 157 (1971), 189-204.

23. On the Suzuki and Conway groups in Representation Theory of Finite Groups and Related

Topics, Proc. Sympos. Pure Math., vol. 21, Amer. Math. Soc., Providence, R.I., 1971, pp. 107-109.

24. S. Mac Lane, Homology, Springer-Verlag, New York, 1963.

25. F. J. MacWilliams and N. J. A. Sloane, The theory of error-correcting codes, North-Holland, Amsterdam, 1981.

26. J. Milnor and D. Husemoller, Symmetric bilinear forms, Springer-Verlag, New York, 1973.

27. H. H. Mitchell, Determination of all primitive collineation groups in more than four variables, Amer. J. Math. 36 (1914), 1-12.

28. H. V. Niemeier, Definite quadratische Formen der Dimension 24 und Diskriminante 1, J. Number Theory 5 (1973), 142-178.

29. S. Norton, $\boldsymbol{A}$ bound for the covering radius of the Leech lattice, Proc. Roy. Soc. London Ser. A 380 (1982), 259-260.

30. O. T. O’Meara, Introduction to quadratic forms, Springer-Verlag, New York, 1971.

31. W. Plesken and M. Pohst, Constructing integral lattices with prescribed minimum. I, preprint.

32. M. Pohst, On integral lattice constructions, Abstracts Amer. Math. Soc. 3 (1982), 152; Abstract \#793-12-14.

33. G. C. Shephard and J. A. Todd, Finite unitary reflection groups, Canad. J. Math. 6 (1954), 274-304.

34. N. J. A. Sloane, Self-dual codes and lattices in Relations Between Combinatorics and Other Parts of Mathematics, Proc. Sympos. Pure Math., vol. 34, Amer. Math. Soc., Providence, R.I., 1979, pp. 273-308.

35. H. Stark, A complete determination of the complex fields of class-number one, Michigan Math. J. 14 (1967), 1-27.

36. J. G. Thompson, private communication.

37. N. M. Vetchinkin, Uniqueness of classes of positive quadratic forms on which values of Hermite constants are attained for $6 \leqslant n \leqslant 8$, Trudy Mat. Inst. Steklov 152 (1980), 34-86. (Russian). English translation in Proc. Steklov Inst. Math. 1982, issue 3, pp. 37-95.

38. G. L. Watson, The number of minimum points of a positive quadratic form, Dissertationes Math. 84 (1971), 42 pages.

39. R. A. Wilson, The complex Leech lattice and maximal subgroups of the Suzuki group, J. Algebra (to appear).

Department of Pure Mathematics and Mathematical Statistics, University of Cambridge, Cambridge CB2 1SB, England

Mathematics and Statistics Research Center, Bell laboratories, Murray Hill, New Jersey 07974 